\title{
HepaCAM-PIK3CA axis regulates the reprogramming of glutamine metabolism to inhibit prostate cancer cell proliferation
}

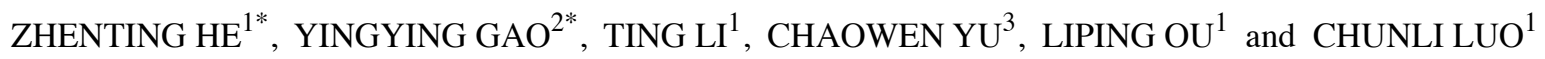 \\ ${ }^{1}$ Key Laboratory of Clinical Laboratory Diagnostics (Ministry of Education), School of Laboratory Medicine, \\ Chongqing Medical University, Chongqing 400016; ${ }^{2}$ Department of Laboratory Diagnosis, People's Hospital of \\ Chongqing Banan District, Chongqing $401320 ;{ }^{3}$ Center for Clinical Molecular Medicine, \\ Children's Hospital of Chongqing Medical University, Chongqing 400014, P.R. China
}

Received August 20, 2021; Accepted February 9, 2022

DOI: $10.3892 / \mathrm{ijo} .2022 .5327$

\begin{abstract}
Energy metabolism reprogramming is becoming an increasingly important hallmark of cancer. Specifically, cancers tend to undergo metabolic reprogramming to upregulate a cell-dependent glutamine (Gln) metabolism. Notably, hepatocellular cell adhesion molecule (HepaCAM) has been previously reported to serve a key role as a tumour suppressor. However, the possible regulatory role of HepaCAM in Gln metabolism in prostate cancer ( $\mathrm{PCa}$ ) remains poorly understood. In the present study, bioinformatics analysis predicted a significant negative correlation among the expression of HepaCAM, phosphatidylinositol-4,5-bisphosphate 3-kinase catalytic subunit $\alpha$ (PIK3CA), glutaminase (GLS) and solute carrier family 1 member 5 (SLC1A5), components of Gln metabolism, in clinical and genomic datasets. Immunohistochemistry results verified a negative correlation between HepaCAM and PIK3CA expression in PCa tissues. Subsequently, liquid chromatography-tandem mass spectrometry (LC-MS/MS) and gas chromatography-mass spectrometry (GC-MS) assays were performed, and the results revealed markedly reduced levels of Gln and metabolic flux in the blood samples of patients with PCa and in PCa cells. Mechanistically, overexpression of HepaCAM inhibited Gln metabolism and proliferation by regulating PIK3CA in $\mathrm{PCa}$ cells. In addition, Gln metabolism was discovered to be stress-resistant in PCa cells, since the expression levels of GLS
\end{abstract}

Correspondence to: Professor Liping Ou or Professor Chunli Luo, Key Laboratory of Clinical Laboratory Diagnostics (Ministry of Education), School of Laboratory Medicine, Chongqing Medical University, 1 Medical College Road, Yuzhong, Chongqing 400016, P.R. China

E-mail: 17783683047@163.com

E-mail: luochunli2021@163.com

${ }^{*}$ Contributed equally

Key words: hepatocellular cell adhesion molecule, phosphatidylinositol-4,5-bisphosphate 3-kinase catalytic subunit $\alpha$, prostate cancer, glutamine metabolic reprogramming, proliferation and SLC1A5 remained high for a period of time after Gln starvation. However, overexpression of HepaCAM reversed this resistance to some extent. Additionally, alpelisib, a specific inhibitor of PIK3CA, effectively potentiated the inhibitory effects of HepaCAM overexpression on Gln metabolism and cell proliferation through mass spectrometry and CCK- 8 experiments. In addition, the inhibitory effect of PIK3CA on the growth of tumor tissue in nude mice was also confirmed by immunohistochemistry in vivo. To conclude, the results from the present study revealed an abnormal Gln metabolic profile in the blood samples of patients with $\mathrm{PCa}$, suggesting that it can be applied as a clinical diagnostic tool for PCa. Additionally, a key role of the HepaCAM/PIK3CA axis in regulating Gln metabolism, cell proliferation and tumour growth was identified. The combination of alpelisib treatment with the upregulation of HepaCAM expression may serve as a novel method for treating patients with PCa.

\section{Introduction}

Prostate cancer $(\mathrm{PCa})$ is one of the most prevalent urological malignancies, with the second-highest rate of cancer-associated mortality among the male population in Western countries (1). Over the past decade, with the rapid growth of an aging population in China, the incidence, morbidity pattern and mortality rates of $\mathrm{PCa}$ have all been increasing on an annual basis. Notably, the majority of patients with PCa develop incurable disease due to advanced regional disease and/or distant metastasis (2).

Cancer cells exhibit the ability to survive and proliferate even under unfavourable conditions, such as nutrient-poor environments (3). Previous reports have demonstrated that energy metabolism reprogramming, which potentiates cell proliferation by adjusting energy metabolism, is an emerging hallmark of cancer $(4,5)$. In particular, metabolic reprogramming towards glutamine (Gln) and cell-dependent Gln metabolic activity have both been found to be increased in a variety of cancers and this is a common metabolic alteration in cancers, where the importance of Gln as a nutrient is considered second only to glucose (5). Gln is one of the most abundant free amino acids and can regulate ATP production, macromolecular synthesis and signal transmission in cancer 
cells by donating its nitrogen and carbon atoms (6). In general, Gln is imported into the cell through specific transporters, such as solute carrier family 1 member 5 (SLC1A5; also known as ASCT2). Gln metabolism begins with its conversion to glutamate (Glu), in a process catalysed by glutaminase (GLS), with type I glutaminase (GLS1) as the dominant isoform expressed in humans $(7,8)$. Additional studies have revealed a close relationship between Gln metabolism and PCa, such that Gln has been proposed to be a potential marker for the diagnosis or treatment of PCa (9-12).

Hepatocellular cell adhesion molecule (HepaCAM) is a novel type of immunoglobulin (Ig) cell adhesion molecule that has shown tumour suppressor-like characteristics and was first isolated from the human liver (13). Under physiological conditions, HepaCAM is expressed at high levels in normal tissues and cells (14). However, the expression of HepaCAM was previously found to be downregulated or even lost in several tumour tissues and cells, including PCa (13-15). In addition, HepaCAM expression has been negatively associated with the proliferation, invasion and the Warburg effect in malignancies of the urinary system, including bladder cancer and renal cell carcinoma (16-19). Overexpression of HepaCAM has also been found to inhibit the proliferation and invasion of PCa cells (20), However, the possible regulatory mechanism of HepaCAM in regards to Gln metabolism in PCa remains poorly understood.

Previous reports have found the PI3K signalling pathway to be one of the most frequently dysregulated pathways in cancer (21-24). Phosphatidylinositol-4,5-bisphosphate 3-kinase catalytic subunit $\alpha$ (PIK3CA) is one of the most common oncogenes which encodes the $110 \alpha$ catalytic subunit of PI3K (25). PIK3CA can be constitutively activated by two main different mechanisms, namely activating mutation and amplification (26). Accumulating evidence has revealed that PIK3CA is associated with Gln metabolism reprogramming in numerous types of cancers (27-30). Consequently, PIK3CA may also be a key regulatory target for reprogramming Gln metabolism in PCa.

The present study explored the possible role of HepaCAM in Gln metabolism in PCa, in addition to attempting the identification of the potential relationship between HepaCAM and PIK3CA expression. It was hypothesized that the overexpression of HepaCAM can inhibit Gln metabolism reprogramming and PCa cell proliferation by regulating PIK3CA.

\section{Materials and methods}

Bioinformatics analysis. A total of 19 PCa datasets (source link: https://www.cbioportal.org/study/summary?id =prad_mich\%2Cprad_su2c_2019\%2Cprad_su2c_2015\%2C prad_mcspc_mskcc_2020\%2Cprad_broad_2013\%2Cprad_ broad\%2Cprad_cpcg_2017\%2Cprad_cdk12_mskcc_2020\% 2Cprad_mskcc\%2Cprad_p1000\%2Cprad_eururol_2017\% 2Cprad_tcga_pan_can_atlas_2018\%2Cprad_mskcc_cheny1_ organoids_2014\%2Cprad_mskcc_2017\%2Cprad_ msk_2019\%2Cprad_tcga_pub\%2Cprad_tcga\%2Cprostate_ dkfz_2018\%2Cmpcproject_broad_2021), including The Cancer Genome Atlas, TCGA (https:// www.cancer.gov/about-nci/organization/ccg/research/structural-genomics/tcga) were downloaded from the cBioPortal (https://www.cbioportal.org). In total, 7 datasets had HepaCAM structural variation, mutation or copy number variation (CNA) data, with 2,093 cases (94.19\%) belonging to PCa. In addition, 19 datasets had PIK3CA structural variation, mutation or CNA data, including 6,671 (95.5\%) cases of PCa (including castration-resistant $\mathrm{PCa}$ and prostate neuroendocrine carcinoma) and 324 cases $(4.5 \%)$ of a normal group $(\mathrm{N})$. The present study was conducted in accordanc with the Declaration of Helsinki (as revised in 2013).

Blood and tissue samples. Between 2016 and 2018, tissue specimens were collected from 17 patients (age range, 53-86 years; mean age, 70) with benign prostatic hyperplasia (BPH) and 44 patients (age range, 55-91 years; mean age, 71) with PCa. In addition, blood specimens were collected from 43 individuals containing normal samples and 66 patients with PCa from 2018 to 2019. All patients were recruited from the Department of Urology, The First Affiliated Hospital of Chongqing Medical University (Chongqing China). All samples were verified by a pathologist, who histologically diagnosed the samples as either $\mathrm{BPH}$ or PCa. The present study was approved by the Ethics Committee of Chongqing University. All patients provided written informed consent prior to specimen acquisition.

Immunohistochemistry. All samples involved in the experiment, including human $\mathrm{PCa}$ and $\mathrm{BPH}$ specimens, were cut into paraffin sections. Detection of HepaCAM and PIK3CA expression in $\mathrm{PCa}$ and $\mathrm{BPH}$ tissue samples was performed using the IHC staining procedure as described previously (31). The primary antibodies used were as follows: anti-HepaCAM (cat. no. MAB4108; 1:150; ProteinTech Group, Inc.) and anti-PIK3CA (cat. no. 4249T; 1:200; Cell Signaling Technology, Inc.). Tissues underwent fixation with $4 \%$ paraformaldehyde, were embedded in paraffin and then subjected to standard dewaxing and rehydration. The sections were incubated in citric acid buffer ( $\mathrm{pH}$ 6.0) for $15 \mathrm{~min}$ for antigen retrieval, followed by incubation for $10 \mathrm{~min}$ with $3 \% \mathrm{H}_{2} \mathrm{O}_{2}$ solution to inactivate endogenous enzymatic activity. The sections were then incubated with anti-HepaCAM antibody and anti-PIK3CA antibody for $1 \mathrm{~h}$ at $25^{\circ} \mathrm{C}$ followed by Elivision Plus Polyer HRP (mouse/rabbit) immunohistochemistry kit (cat. no. KIT-9903; 1:1,000; Maxim Biotech, Inc.) for $30 \mathrm{~min}$ at $20^{\circ} \mathrm{C}$. The results of HepaCAM and PIK3CA expression in $\mathrm{PCa}$ and $\mathrm{BPH}$ tissue samples was analysed according to a previously described procedure (31).

Cell culture and treatment. Prostate cell lines PC3 (cat. no. ZQ0041), LNCaP (cat. no. ZQ0039) and RWPE-1 (cat. no. ZQ0351) were obtained from Shanghai ZhongQiao XinZhou Biotechnology Co., Ltd. RWPE-1 cells were cultured with Keratinocyte-SFM medium (cat. no. 10744-019; Gibco; Thermo Fisher Scientific, Inc.), whereas cancer cell lines PC3 and LNCaP cells were cultured with RPMI-1640 media (Gibco; Thermo Fisher Scientific, Inc.) supplemented with 10\% FBS (Gibco; Thermo Fisher Scientific, Inc.) and $100 \mathrm{U} / \mathrm{ml}$ penicillin/streptomycin (Beyotime Institute of Biotechnology). All cells were cultured in a $37^{\circ} \mathrm{C}$ incubator containing $5 \% \mathrm{CO}_{2}$ and $1 \% \mathrm{O}_{2}$ with $45-65 \%$ humidity. Adenoviruses encoding HepaCAM (Ad-HepaCAM) were stored at $-80^{\circ} \mathrm{C}$ and amplified in $293 \mathrm{~A}$ cells. The viral fluid was obtained after the repeated freezing and thawing of the 293A cells. The prostate cancer cells were transfected with either Ad-HepaCAM or Ad-GFP, 
respectively. Cells (1x10\%/well) were seeded into 6-well plates and incubated with $2 \mathrm{ml}$ complete medium for $12 \mathrm{~h}$, before being transfected with adenoviruses and/or alpelisib $(8 \mu \mathrm{M}$, $24 \mathrm{~h}$; MedChemExpress, USA). After 24 or $72 \mathrm{~h}$ of incubation, subsequent experiments were performed.

Gln deprivation experiments. After the cells had completely attached to the culture dish, the RPMI-1640 medium was removed and PC3 and LNCaP cells were washed once with PBS. Gln-free medium was prepared using RPMI-1640 devoid of Gln (cat. no. A14431; Thermo Fisher Scientific, Inc.) but other nutrients were retained at the same concentrations as those in the standard complete RPMI-1640 media. Complete RPMI-1640 medium was prepared by supplementing with 2 mM L-Gln (Beijing Solarbio Science \& Technology Co., Ltd.). All media were supplemented with $10 \%$ dialyzed FBS (Gemini Bio Products) and $100 \mathrm{U} / \mathrm{ml}$ penicillin/streptomycin. All cells were cultured in a $37^{\circ} \mathrm{C}$ incubator containing $5 \%$ $\mathrm{CO}_{2}$ and $1 \% \mathrm{O}_{2}$ with $45-65 \%$ humidity. The incubation time depended on the experimental requirements (24-72 h).

Reverse transcription-quantitative polymerase chain reaction (RT-qPCR). RNA was extracted from RWPE-1, PC3 and LNCaP cells using TRIzol (Thermo Fisher Scientific, Inc.), and PrimeScript RT reagent kits (Takara Bio, Inc.) were used to reverse transcribe the RNA. Real-time PCR was performed with the SYBR Premix Ex Taq ${ }^{\mathrm{TM}}$ II kit (cat. no. RR820A; TaKaRa, Inc.). Notably, $\beta$-actin was used as the standard reference. The thermocycling conditions for qPCR were as follows: initial denaturation at $95^{\circ} \mathrm{C}$ for $3 \mathrm{~min}$; followed by 40 cycles of $95^{\circ} \mathrm{C}$ for $10 \mathrm{sec}, 60^{\circ} \mathrm{C}$ for $20 \mathrm{sec}$ and $72^{\circ} \mathrm{C}$ for $20 \mathrm{sec}$; and final extension at $72^{\circ} \mathrm{C}$ for $5 \mathrm{~min}$. The comparative $2^{-\Delta \Delta \mathrm{Cq}}$ method was used to calculate the relative expression level of mRNA (32). Each experiment was repeated $\geq 3$ times. The primer sequences (5'-3') used were as follows: HepaCAM forward, TGTACAGCTGCATGGTGGAGA and reverse, TCT GGTTTCAGGCGGTCATCA; PIK3CA forward, CCACGAC CATCATCAGGTGAA and reverse, CCTCACGGAGGCATT CTAAAGT; $G L S$ forward, TACTGAGCCCTGAAGC and reverse, TCCAGAGGAGGAGACC; SLC1A5 forward, GAG CTGCTTATCCGCTTCTTC and reverse, GGGGCGTAC CACATGATCC; Cyclin Dl forward, GCTGGAGCCCGT GAAAAAGA and reverse, CTCCGCCTCTGGCATTTTG; proliferating cell nuclear antigen $(P C N A)$ forward, TCAAGA AGGTGTTGGAGGCA and reverse, CAGCGGTAGGTG TCGAAGC and $\beta$-actin forward, TGACGTGGACATCCG CAAAG and reverse, CTGGAAGGTGGACAGCGAGG.

Western blotting. Total proteins were extracted by RIPA buffer (cat. no. 9806; Cell Signaling Technology, Inc.) containing the protease inhibitor phenyl methane sulfonyl fluoride and phosphatase inhibitors $(33,34)$. Nuclear and plasma proteins were extracted using the nuclear and cytoplasmic protein extraction reagent (Beyotime Institute of Biotechnology). The BCA kit (Beyotime Institute of Biotechnology) was used to measure protein concentration. SDS-PAGE (10\% gel) was used for protein separation $(50 \mu \mathrm{g})$. The following antibodies were used: anti-HepaCAM (cat. no. 18177-1-AP; 1:1,000; ProteinTech Group, Inc.), anti-PIK3CA (cat. no. 4249T; 1:1,000; Cell Signaling Technology, Inc.), anti-AKT1 (cat. no. abs131788; 1:1,000; Absin Bioscience, Inc.), anti-phosphorylated (p-)AKT1 (Ser473; cat. no. abs130002; 1:1,000; Absin Bioscience, Inc.), anti-p-AKT1 (Thr308; cat. no. abs130889; 1:1,000; Absin Bioscience, Inc.), anti-GLS1 (cat. no. 56750; 1:1,000, Cell Signaling Technology, Inc.), anti-SLC1A5 (cat. no. 20350-1-AP; 1:3,000; ProteinTech Group, Inc.), anti-cyclin D1 (cat. no. 55506; 1:500, Cell Signaling Technology, Inc.), anti-PCNA (cat. no. 13110; 1:500; Cell Signaling Technology, Inc.), anti- $\beta$-actin (cat. no. 20536-1-AP, 1:5,000; ProteinTech Group, Inc.), and

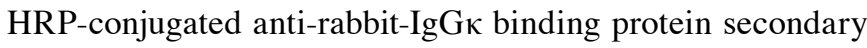
antibody (cat. no. ab205718; 1:5,000; Abcam). Enhanced Chemiluminescence Detection Kit was utilized for the visualization of protein bands (Suzhou New Saimei Biotechnology Co., Ltd.).

Cell Counting Kit-8 (CCK-8) assay. PC3 and LNCaP cells (3x10 $3 /$ well) were first cultured in 96-well plates overnight before being transfected/treated with Ad-HepaCAM/-Gln/alpelisib or control. After $0,24,48$ or $72 \mathrm{~h}, 10 \mu \mathrm{l}$ CCK-8 reagent (cat. no. 354550 Shanghai Univ Biotechnology Co., Ltd.) was added and incubated for $1-4 \mathrm{~h}$ at $37^{\circ} \mathrm{C}$. Finally, absorbance in each well was measured at $450 \mathrm{~nm}$. A total of four wells were used for each treatment group per experiment.

Colony formation assay. PC3 and LNCaP cells (500/well) following blank treatment and administration of Ad-GFP or Ad-HepaCAM were added into 6-well plates and cultured in a $37^{\circ} \mathrm{C}$ incubator containing $5 \% \mathrm{CO}_{2}$ and $1 \% \mathrm{O}_{2}$ with $45-65 \%$ humidity. for 7-14 days. The cells were then fixed with methanol for $2 \mathrm{~min}$ and stained with $0.5 \%$ crystal violet for $10 \mathrm{~min}$ at room temperature and photographed for cell counting. The images of each well were scanned using an Epson scanner GT-X970 (Seiko Epson Corp.), and the colonies were counted using ImageJ $1.52 \mathrm{v}$ software (National Institutes of Health). Each treatment group used three wells per experiment and each experiment was repeated three times independently.

Flow cytometry. PC3 and LNCaP cells were transferred into 6-well plates overnight and transfected/treated with Ad-HepaCAM or alpelisib. After $72 \mathrm{~h}$, the cells were washed two to three times with PBS, before being digested using $0.25 \%$ trypsin, washed twice again with PBS and transferred into Eppendorf tubes. The cells were counted and $1 \times 10^{6}$ cells were resuspended with $100 \mu \mathrm{l}$ PBS before $500 \mu \mathrm{l}$ cooled $75 \%$ ethanol was added after centrifugation. Cell cycle distribution was detected using the CytoFLEX Flow Cytometer (Beckman Coulter, Inc.) and the results were analysed using ModFitLT 5 software (Verity Software House, Inc.).

Immunofluorescence. PC3 and LNCaP cells $\left(3 \times 10^{4}\right)$ were cultured on coverslips in 24-well plates following administration of Ad-HepaCAM or/and alpelisib, and then the cells were incubated for $72 \mathrm{~h}$. The cells were then fixed with $4 \%$ paraformaldehyde, $0.1-1 \%$ Triton X-100 and 5\% goat blocking serum in sequence as previously described (35). Primary antibodies against anti-Ki67 (cat. no. ab197234; 1:100, Abcam), secondary antibodies and 4,6-diamidino-2-pheny-lindole (Zhongshan Golden Bridge Biotechnology, Ltd.; OriGene Technologies, Inc.) were used for incubation with the cells. 
Fluorescence images were captured using a confocal laser scanning microscope (Nikon Corp.) at x400 magnification. The Ki-67-positive index was analysed by Image J (v4.6.2, National Institutes of Health).

Liquid chromatography-tandem mass spectrometry (LC-MS/MS) and gas chromatography-mass spectrometry (GC-MS) assay. The trypsin-digested PC3 and LNCaP cells $\left(>1 \times 10^{7}\right)$ from the different treatment groups were collected, before the supernatant was discarded after centrifugation. Cells were washed three times with PBS before being fully disrupted using a sonicator $(16,000 \mathrm{x}$ g). Finally, pre-cooled methanol was added for fixation. After fixation, the lysed cells were suspended on a dried blood spot (DBS) to be sufficiently saturated. Fresh blood samples from different patients were collected, prepared in a DBS format on a filter paper and stored at $4^{\circ} \mathrm{C}$. MS/MS analysis was achieved using a QTRAP triple quadrupole mass spectrometer (AB SCIEX 3200; AB SCIEX, USA) coupled to a LC system (Shimadziu 20ADXR; Shimadziu).

Approximately $20 \mu \mathrm{l}$ of working solution was directly injected for analysis without prior chromatographic separation. A mixed liquor (acetonitrile: $\mathrm{H}_{2} \mathrm{O}=1: 1$ ) containing $0.05 \%$ FA was used as the mobile phase. The mass spectrometer was equipped with an electrospray ionization (ESI) and operated in the positive ion mode to monitor the $\mathrm{m} / \mathrm{z}$ transitions for all peptides and IS. For each peptide, two multiple reaction monitorings (MRMs) were simultaneously monitored. A scheduled MRM acquisition method was constructed using manually optimized declustering potentials (DP), collision energies (CE), collision cell entrance (CEP) and exit potentials (CXP). All chromatograms were analysed with Analyst 1.5 software (AB SCIEX, USA) using internal standards, and the peak-area ratios were applied for further calculations (36).

Xenograft model in vivo. A total of 10 male nude mice (4 weeks of age; body weight, 10-15 g) were purchased from the Shanghai SLAC Laboratory Animal Co., Ltd. All rats were housed at $23-25^{\circ} \mathrm{C}$ with $50-60 \%$ humidity, 12-h light/dark cycle and food and water ad libitum. The present study was performed in accordance with the Animal Research Advisory Committee (ARAC) Guidelines (https:/oacu.oir.nih.gov/animal-research-advisory-committee-guidelines) and was approved by the Ethics Committee of Chongqing Medical University (Chongqing, China). A prostate cancer xenograft model was established by the subcutaneous injection of LNCaP cells into the right flank of 10 mice $\left(5 \times 10^{6}\right.$ cell per mouse). These nude mice were then randomly divided into a dosing group and control group. Alpelisib was dissolved in $0.5 \%$ sodium carboxyl methyl cellulose (CMC-NA). Alpelisib (12.5 mg/kg) was administered daily through an oral gavage for 5 consecutive days, and then 1 day off and the above process was repeated once. The same amount of $0.5 \%$ CMC-NA was given by intragastric administration to the control group. Tumour size was assessed every day. After 4 weeks, the 10 mice were sacrificed by $\mathrm{CO}_{2}$ euthanasia. The criteria for determining the end point of this animal experiment were as follows: i) the animal was dying or unable to move, or had no response after giving gentle stimulation; ii) difficulty breathing, with typical symptoms being salivation and/or cyanosis in the mouth and nose; iii) diarrhea or urinary incontinence; iv) the body weight was reduced by $20 \%$ of the body weight during the experiment; v) inability to eat or drink; vi) the animal was showing clear signs of anxiety, irritability; vii) the weight of the tumour reached $>10 \%$ of the animal's own body weight; viii) animal skin damage area accounted for $>30 \%$ of the whole body; and ix) or infection and suppuration occurred. $\mathrm{CO}_{2}$ euthanasia was performed using a $\mathrm{CO}_{2}$ delivery system according to the ARAC guide (https://oir.nih.gov/sourcebook/committees-advisory-ddir/animal-research-advisory-committee-arac) for the experimental procedure. The mouse was first placed in the box full of air before the box was filled with a volume of 2 liters $\mathrm{CO}_{2}$ to a volume of 10 liters at a constant rate (the volume displacement rate of $\mathrm{CO}_{2}$ was $60 \% \mathrm{vol} / \mathrm{min}$ ). Each mouse was checked for the lack of breathing and constriction of pupil size. After the breathing halted, the $\mathrm{CO}_{2}$ flow was maintained for $1 \mathrm{~min}$ and the mouse was removed from the euthanasia box. Death was verified by the cessation of heartbeat and breathing, in addition to the reflexes disappearing. Tumour tissues were then isolated for comparing the size and weight before IHC experiments were performed. Tumour volume was calculated using the formula $\mathrm{V}=\pi / 6 \times \mathrm{L} \times \mathrm{W}$, where $\mathrm{V}$ was the volume and $\mathrm{L}$ was the length and $\mathrm{W}$ was the width. The maximum volume and the maximum diameter of a single tumour presented in the present study were $764.5 \mathrm{~mm}^{3}$ and $12.1 \mathrm{~mm}$, respectively.

Statistical analysis. SPSS (version 24.0; IBM Corp.) and GraphPad Prism (version 5; GraphPad Software, Inc.) were used for statistical analysis. Data are presented as the mean \pm SD. All experiments were repeated at least three times. Comparisons between two groups, other than comparisons between tumour and adjacent non-tumour samples, were analysed using the unpaired Student's t-test. A paired Student's t-test was used to compare HepaCAM and PIK3CA expression levels between tumour and adjacent non-tumour samples. Survival curves were assessed using log-rank tests. Comparisons among multiple groups were analysed using one-way ANOVA followed by Tukey's post hoc test. The $\chi^{2}$ test was used to analyse the relationship between HepaCAM and PIK3CA expression or the concentration of Gln/Glu and each of the clinical parameters. The correlation between HepaCAM and PIK3CA expression in the tissue samples was evaluated using Spearman's rank correlation coefficient. $\mathrm{P}<0.05$ was considered to indicate a statistically significant difference.

\section{Results}

Bioinformatics prediction of the correlation among HepaCAM, PIK3CA and Gln metabolism in clinical and genomic datasets. In a preliminary experiment, a correlation between the expression of HepaCAM and GLS was found in PCa cells, but the possibility of direct action was ruled out. The selection of PIK3CA for the present study was based on preliminary bioinformatics analysis, where protein interaction analysis in the STRING protein database found a possibility of protein interaction between HepaCAM and PIK3CA. In addition, previous studies have found that the overexpression of HepaCAM was able to inhibit the expression of c-Myc $(37,38)$, which is an important regulatory target of SLC1A5 (39). Under these premises, the correlation among these components aforementioned was assessed through bioinformatics analysis. Comparative analysis of the different datasets in the 

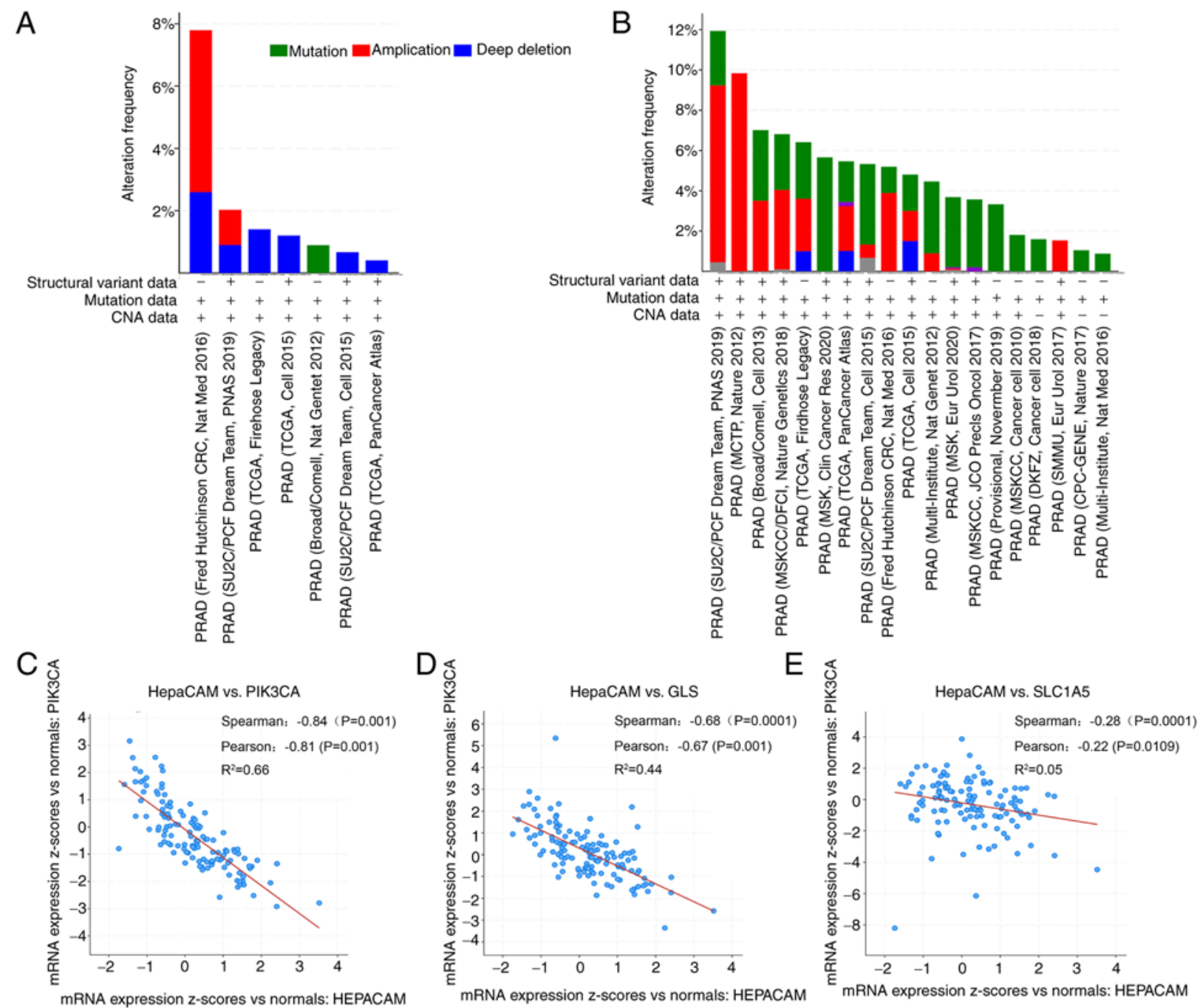

$\mathrm{F}$

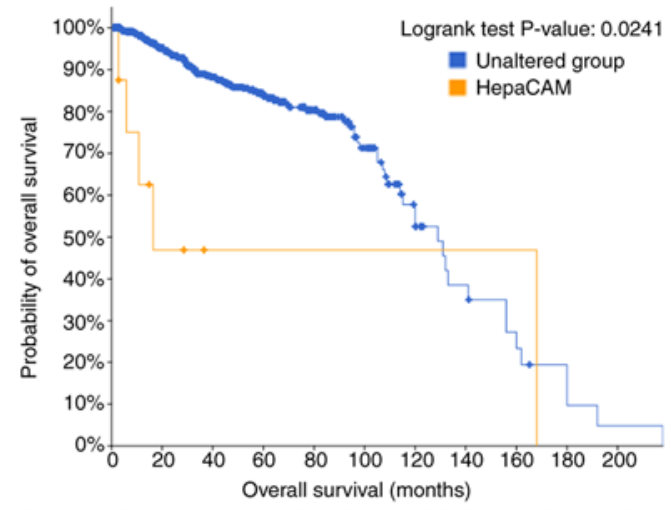

Number of cases, total Number of events Median months overall $(95 \% \mathrm{Cl})$ $\begin{array}{rccc}\text { Unaltered group } & 926 & 129 & 129.00(115.13-156.00) \\ \text { HepaCAM } & 8 & 5 & 16.43(10.74-\mathrm{NA})\end{array}$

\section{G}

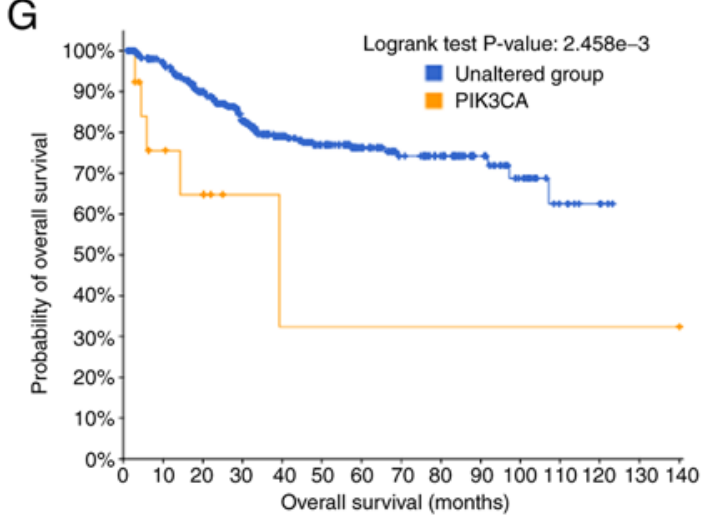

Number of cases, total Number of events Median months overall $(95 \% \mathrm{Cl})$ $\begin{array}{llll}\text { Unaltered group } & 422 & 79 & \text { NA }\end{array}$

Figure 1. The correlation and difference between HepaCAM, PIK3CA, and glutamine metabolism-related molecules from the Bio-databases (A and B) Variation types and proportion of HepaCAM and PIK3CA genes in different PCa datasets from the cBioportal. (C-E) RNA-seq mRNA expression data from GEO database (GSE21032) was used to compare the correlation of HepaCAM with PIK3CA, GLS and SLC1A5 in PCa tumors. (F and G) Overall survival curves of PCa patients according to HepaCAM and PIK3CA mRNA levels in TCGA. HepaCAM, hepatocellular cell adhesion molecule; PIK3CA, phosphatidylinositol-4,5-bisphosphate 3-kinase catalytic subunit $\alpha$; GLS, glutaminase; SLC1A5, solute carrier family 1 member 5. To note: the link to the 19 PCa datasets is provided in the Bioinformatics analysis section.

cBioportal database revealed little to no HepaCAM expression in the majority of cancer types tested (Fig. 1A). Furthermore, the main alteration in PIK3CA expression in PCa was found to be amplification (Fig. 1B), while mutation was the major form of alteration in other solid tumours (40,41). Additionally, the co-expression analysis of genes in the databases found that the mRNA expression levels of HepaCAM exhibited a negative correlation with those of PIK3CA and components 
Table I. Glutamine/glutamate concentrations and clinicopathological parameters of the PCa patients.

\begin{tabular}{|c|c|c|c|c|c|c|c|}
\hline \multirow[b]{3}{*}{ Characteristics } & \multirow{3}{*}{$\begin{array}{c}\text { No. of } \\
\text { specimens (\%) }\end{array}$} & \multicolumn{3}{|c|}{ Glutamine $(\mathrm{Gln})(\mu \mathrm{mol} / \mathrm{l})$} & \multicolumn{3}{|c|}{ Glutamate (Glu) $(\mu \mathrm{mol} / \mathrm{l})$} \\
\hline & & \multicolumn{3}{|c|}{ Median=9.766 } & \multicolumn{3}{|c|}{ Median=66.613 } \\
\hline & & $<9.766$ & $\geq 9.766$ & $\mathrm{P}$-value & $<66.613$ & $\geq 66.613$ & P-value \\
\hline \multicolumn{8}{|l|}{ Histology } \\
\hline Normal & 43 & 0 & 43 & 0.001 & 41 & 2 & 0.001 \\
\hline $\mathrm{PCa}$ & 66 & $47(71)$ & $19(29)$ & & $13(20)$ & $53(80)$ & \\
\hline \multicolumn{8}{|l|}{ Age (years) } \\
\hline$<60$ & $9(14)$ & $4(6)$ & $5(8)$ & 0.056 & $1(2)$ & $8(12)$ & 0.486 \\
\hline$\geq 60$ & $57(86)$ & $43(65)$ & $14(21)$ & & $12(18)$ & $45(68)$ & \\
\hline \multicolumn{8}{|l|}{$\operatorname{PSA}(\mu \mathrm{g} / \mathrm{l})$} \\
\hline \multicolumn{8}{|l|}{ Median $=20.67$} \\
\hline$<20.67$ & $31(47)$ & $25(38)$ & $6(9)$ & 0.233 & $4(6)$ & $27(41)$ & 0.192 \\
\hline$\geq 20.67$ & $35(53)$ & $22(33)$ & $13(20)$ & & $9(14)$ & $26(39)$ & \\
\hline \multicolumn{8}{|c|}{ Gleason score of $\mathrm{PCa}$} \\
\hline$\leq 6$ & $19(29)$ & $10(15)$ & $9(14)$ & & $5(8)$ & $14(21)$ & \\
\hline 7 & $23(35)$ & $15(23)$ & $8(12)$ & 0.014 & $4(6)$ & $19(29)$ & 0.690 \\
\hline$\geq 8$ & $24(36)$ & $22(33)$ & $2(3)$ & & $4(6)$ & $20(30)$ & \\
\hline
\end{tabular}

Mann-Whitney test for 2 independent variables; Chi-square test for trend for the number of rows or columns $>2$. Numbers in bold font indicate statistical significance $(\mathrm{P}<0.05)$. $\mathrm{PCa}$, prostate cancer; PSA, prostate-specific antigen.

Table II. HepaCAM and PIK3CA in PCa tissues and clinicopathological parameters.

\begin{tabular}{|c|c|c|c|c|c|c|c|}
\hline \multirow[b]{2}{*}{ Characteristics } & \multirow{2}{*}{$\begin{array}{c}\text { No. of } \\
\text { specimens }(\%)\end{array}$} & \multicolumn{3}{|c|}{ HepaCAM staining } & \multicolumn{3}{|c|}{ PIK3CA staining } \\
\hline & & Positive & Negative & P-value & Positive & Negative & P-value \\
\hline Total & $44(100)$ & $15(34)$ & $29(66)$ & & $38(86)$ & $6(14)$ & \\
\hline \multicolumn{8}{|l|}{ Age (year) } \\
\hline$<60$ & $16(36)$ & $7(16)$ & $4(20)$ & 0.307 & $13(29)$ & $3(7)$ & 0.052 \\
\hline$\geq 60$ & $28(64)$ & $8(18)$ & $20(46)$ & & $25(57)$ & $3(7)$ & \\
\hline \multicolumn{8}{|c|}{ Histological stage } \\
\hline Ta-T2 & $29(66)$ & $10(23)$ & $19(43)$ & 0.939 & $27(61)$ & $2(5)$ & 0.07 \\
\hline $\mathrm{T} 3-\mathrm{T} 4$ & $15(34)$ & $5(11)$ & $10(23)$ & & $11(25)$ & $4(9)$ & \\
\hline \multicolumn{8}{|l|}{ Gleason score } \\
\hline$<6$ & $10(23)$ & $8(18)$ & $2(5)$ & & $6(14)$ & $4(9)$ & \\
\hline 7 & $16(36)$ & $7(16)$ & $9(20)$ & 0.001 & $15(34)$ & $1(2)$ & 0.022 \\
\hline$\geq 8$ & $18(41)$ & $0(0)$ & $18(41)$ & & $17(38)$ & $1(3)$ & \\
\hline
\end{tabular}

Mann-Whitney test for 2 independent variables; Chi-square test for trend for the number of rows or columns $>2$. Numbers in bold font indicate statistical significance $(\mathrm{P}<0.05)$. HepaCAM, hepatocellular cell adhesion molecule; PCa, prostate cancer; PIK3CA, phosphatidylinositol-4,5-bisphosphate 3-kinase catalytic subunit $\alpha$.

associated with Gln metabolism, GLS and SLC1A5 $(\mathrm{P}<0.05$; Fig. 1C-E). Survival analyses of the datasets found that the patients whose samples possessed lower expression levels of HepaCAM displayed poorer overall survival $(\mathrm{P}<0.01$; Fig. $1 \mathrm{~F})$, whereas the survival trend for PIK3CA expression was opposite $(\mathrm{P}<0.05$; Fig. 1G).
Abnormal levels of Gln in blood samples and PCa cell lines. Blood samples were collected from 66 patients with PCa and 43 normal individuals for the detection of amino acid concentrations by LC-MS/MS and GC-MS testing. Among the 15 common amino acids detected, only the levels of Gln and arginine (Arg) in the blood samples of patients with PCa were 


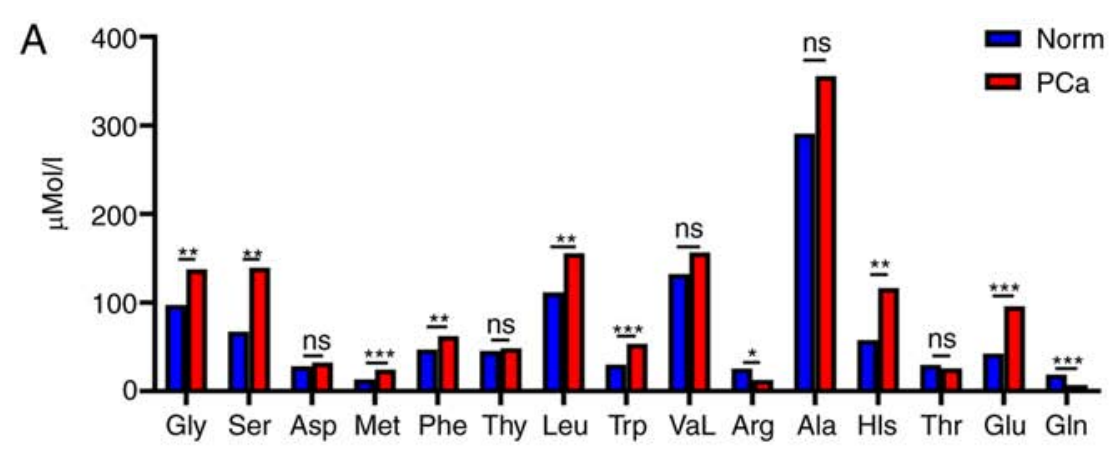

B

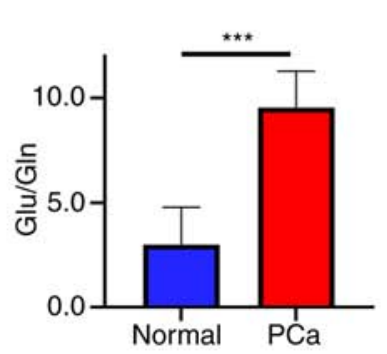

E

$H \& E$

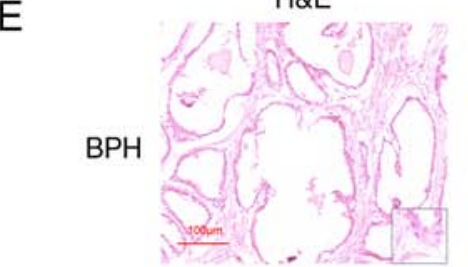

F

G

MGPCa

$\mathrm{H}$

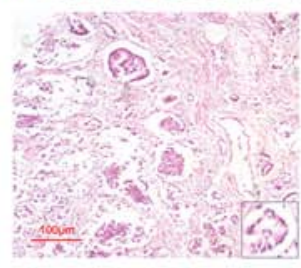

HGPCa
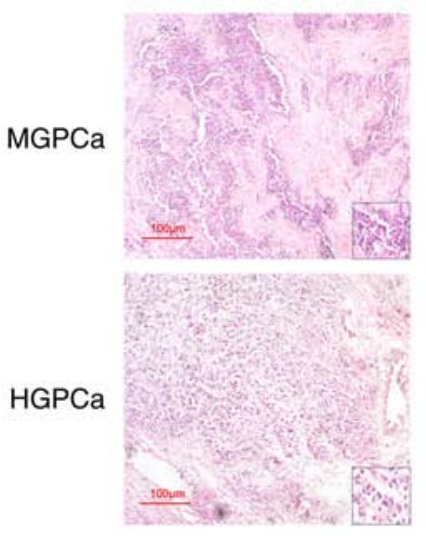

C

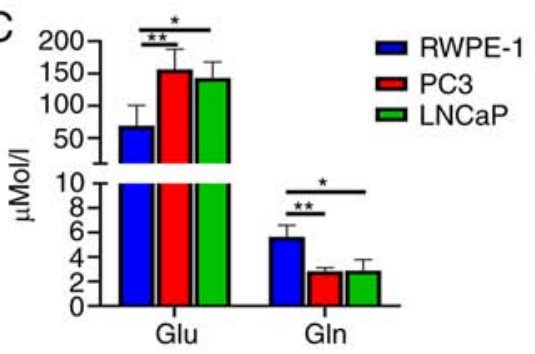

HepaCAM
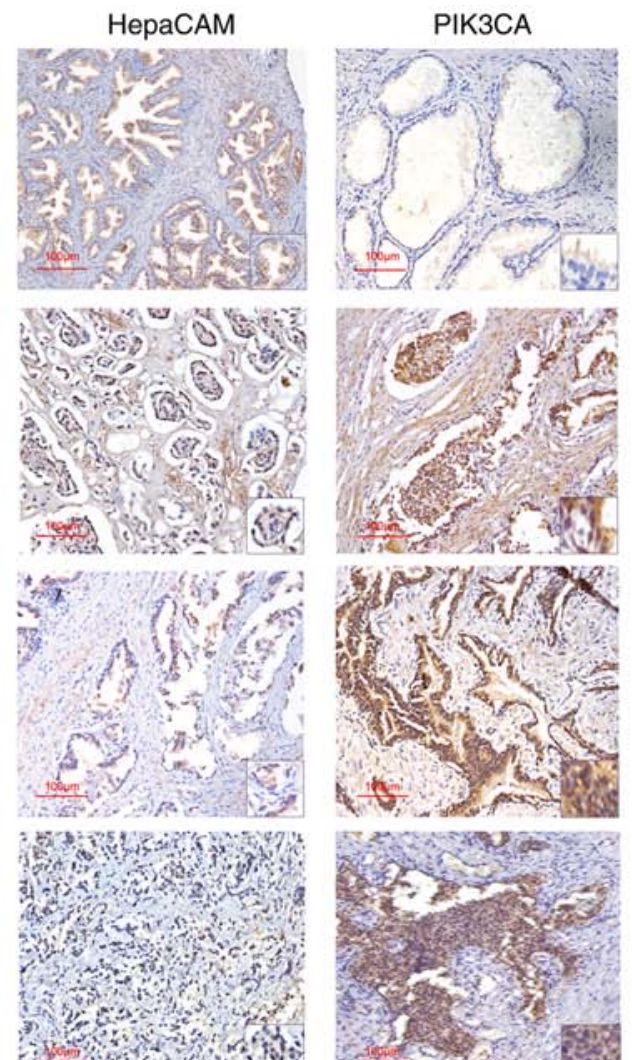

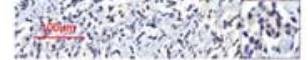
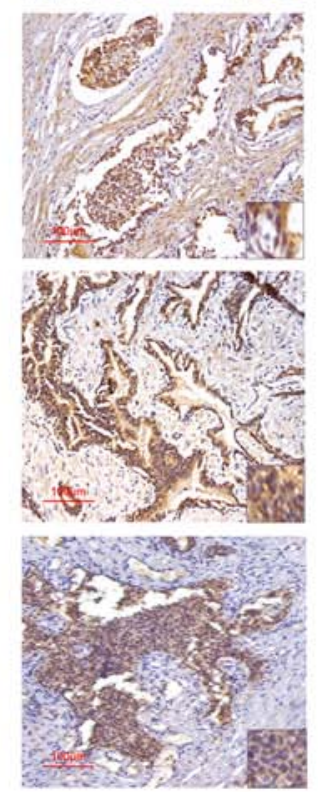
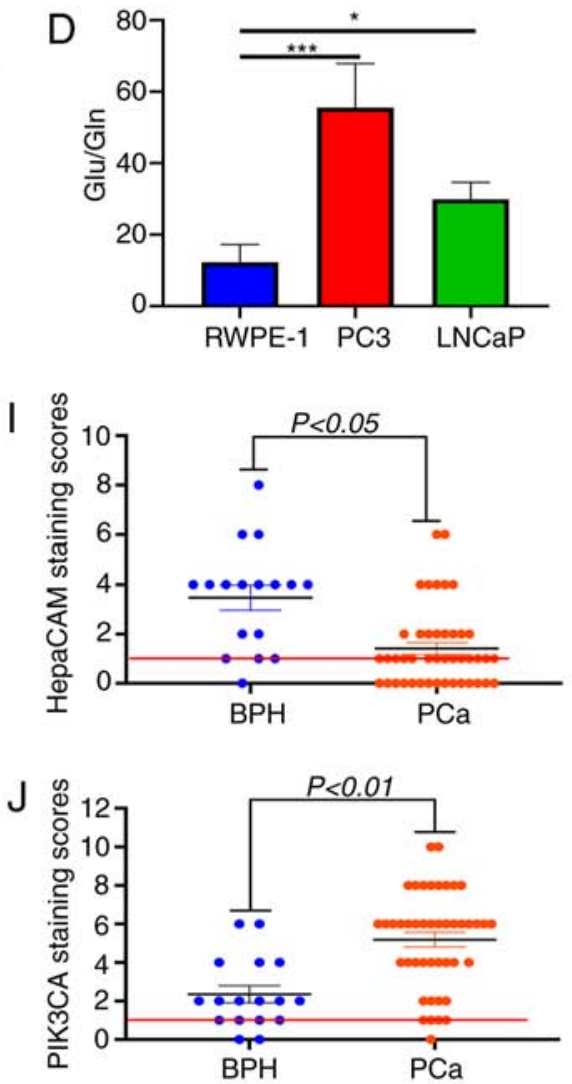

K

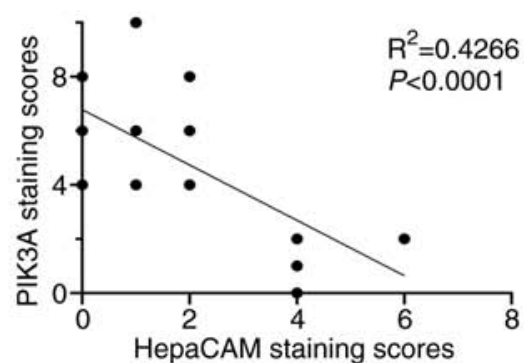

Figure 2. The significant difference in glutamine (Gln)/glutamate (Glu) levels in blood specimens or cells and HepaCAM and PIK3CA expression in tissues specimen. (A) Multiple common amino acid concentrations in different blood samples tested by mass spectrometry and analysed by Mann-Whitney test. ${ }^{*} \mathrm{P}<0.05,{ }^{* *} \mathrm{P}<0.01,{ }^{* * * *} \mathrm{P}<0.001$; ns, not significant comparing PCa vs. normal (Norm) specimens. (B) Comparison of the ratio of Glu to Gln between PCa and normal samples. ${ }^{* * *} \mathrm{P}<0.001$ vs. normal specimens. (C and D) Difference in the concentration of Glu and Gln between prostate cancer cell lines PC3 and LNCaP and normal epithelial RWPE-1 cells. ${ }^{*} \mathrm{P}<0.05,{ }^{* *} \mathrm{P}<0.01,{ }^{* * *} \mathrm{P}<0.001$. (E-H) Representative hematoxylin and eosin (H\&E) staining and IHC staining for HepaCAM and PIK3CA in PCa and BPH samples, Magnification, x200. (I and J) Staining scores of HepaCAM and PIK3CA expression in BPH and PCa tissues. (K) Correlation analysis between HepaCAM and PIK3CA in tissues as analysed by Spearman's correlation analysis. Glu, glutamate; Gln, glutamine; LGPCa, low-grade PCa; MGPCa, middle-grade PCa; HGPCa, high-grade PCa; BPH, benign prostatic hyperplasia; PCa, prostate cancer; HepaCAM, hepatocellular cell adhesion molecule; PIK3CA, phosphatidylinositol-4,5-bisphosphate 3-kinase catalytic subunit $\alpha$.

significantly lower compared with those in the samples from the normal individuals ( $\mathrm{P}<0.05$; Fig. $2 \mathrm{~A}$; Table $\mathrm{I}$ ). In addition, the concentration of Gln exhibited the most striking difference among all other amino acids tested, where the main metabolic flow was towards the synthesis of glutamic acid $(\mathrm{P}<0.001$; Fig. 2A and B; Table I). 
A

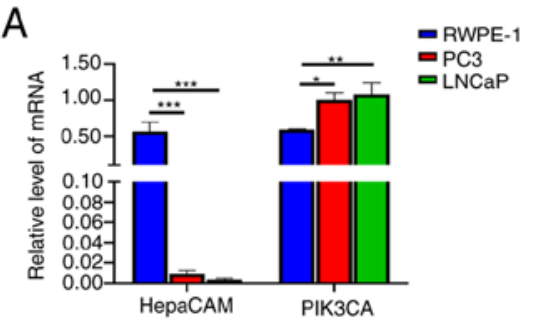

C
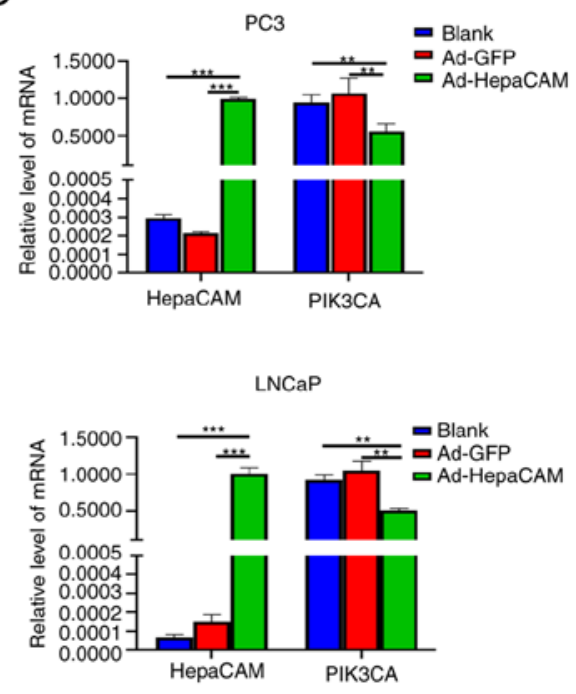

B
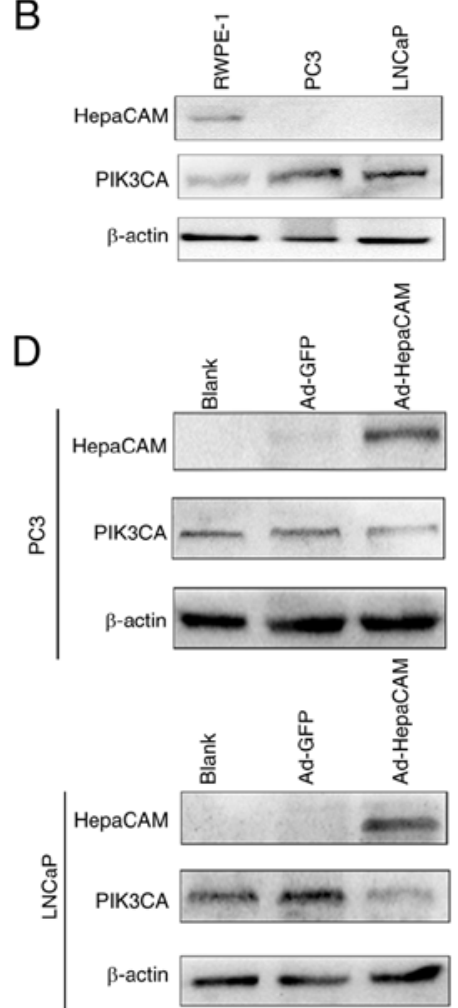

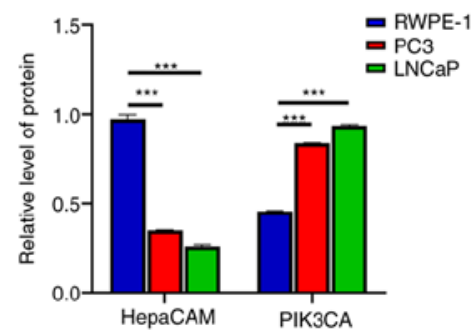

E
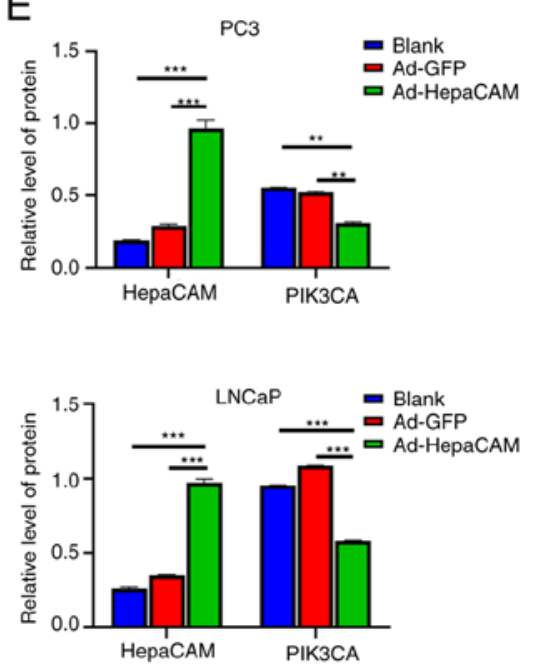
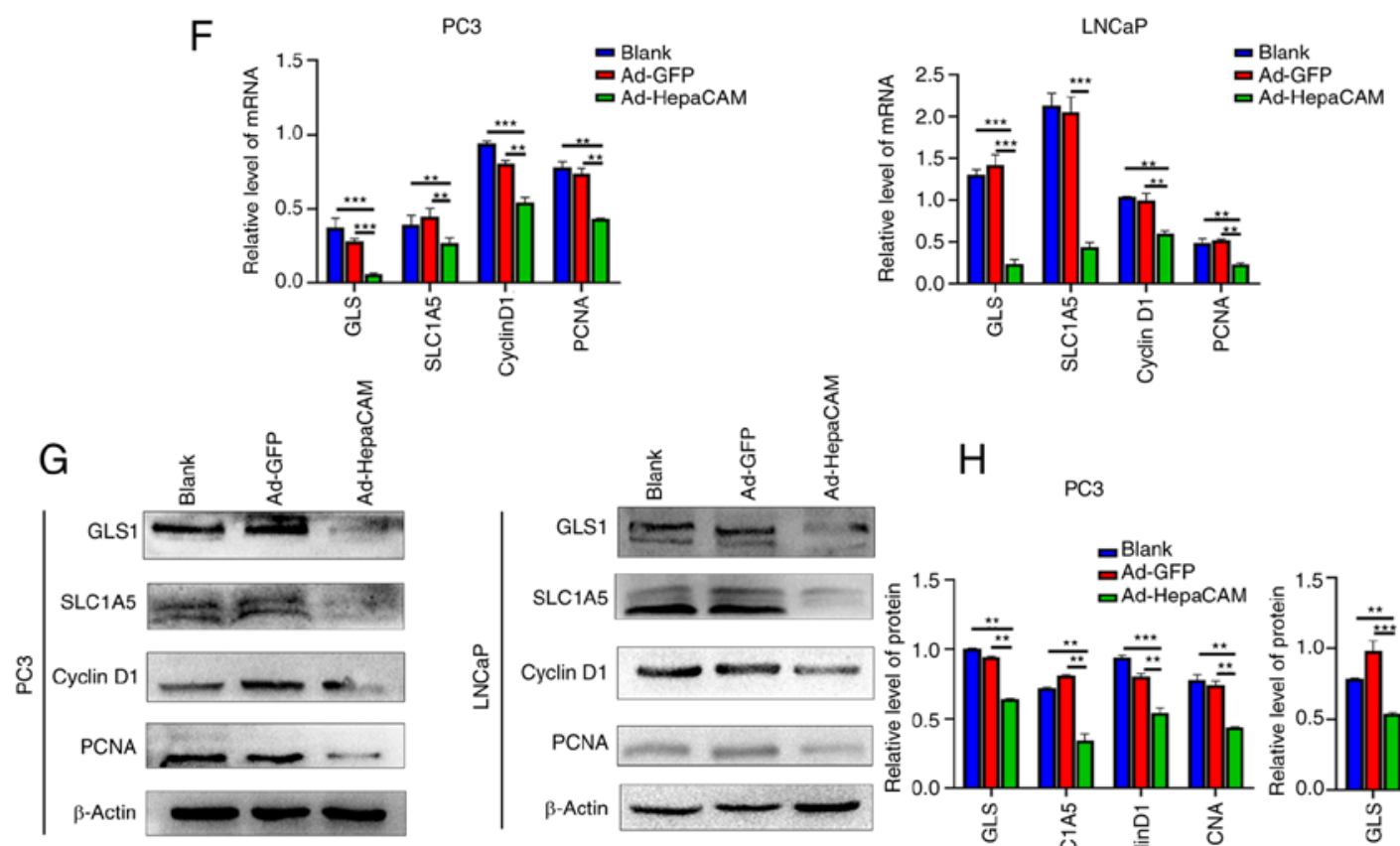

$\mathrm{H}$

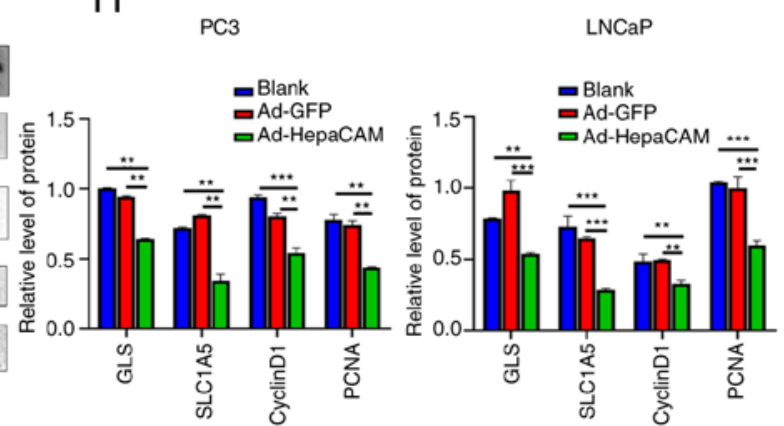

Figure 3. Overexpression of HepaCAM inhibits the expression of PIK3CA and glutamine metabolism or proliferation related molecules in PCa. (A and B) Expression of HepaCAM and PIK3CA in RWPE-1, PC3 and LNCaP cells was detected by (A) RT-qPCR and (B) western blot analysis. "P<0.05, ${ }^{* *} \mathrm{P}<0.01$, and ${ }^{* * *} \mathrm{P}<0.001$ compared with the RWPE-1 cells. (C-E) Overexpression of HepaCAM adenovirus and corresponding changes in PIK3CA at the (C) mRNA and (D and E) protein levels was verified in PC3 and LNCaP cells. (F-H) mRNA and protein levels of GLS, SLC1A5, cyclin D1 and PCNA in cells were detected by qPCR and western blot analysis after infection with adenoviral Ad-HepaCAM. $\beta$-actin was used as internal controls. Data are represented as mean $\pm \mathrm{SD}$ of 3 individual experiments. ${ }^{* *} \mathrm{P}<0.01$ and ${ }^{* * * *} \mathrm{P}<0.001$ vs. blank control. HepaCAM, hepatocellular cell adhesion molecule; PIK3CA, phosphatidylinositol-4,5-bisphosphate 3-kinase catalytic subunit $\alpha$; GLS, glutaminase; SLC1A5, solute carrier family 1 member 5; PCNA, proliferating cell nuclear antigen.

On a cellular level, the Gln levels in RWPE-1 cells was significantly higher compared with that in $\mathrm{LNCaP}$ and $\mathrm{PC} 3$ cells $(\mathrm{P}<0.01$; Fig. $2 \mathrm{C})$, where the metabolic flux was also biased towards synthesizing Glu ( $\mathrm{P}<0.001$; Fig. 2D). Additionally, high levels of Gln in PCa were found to be significantly associated with the Gleason score (Table I). These results suggest 
A

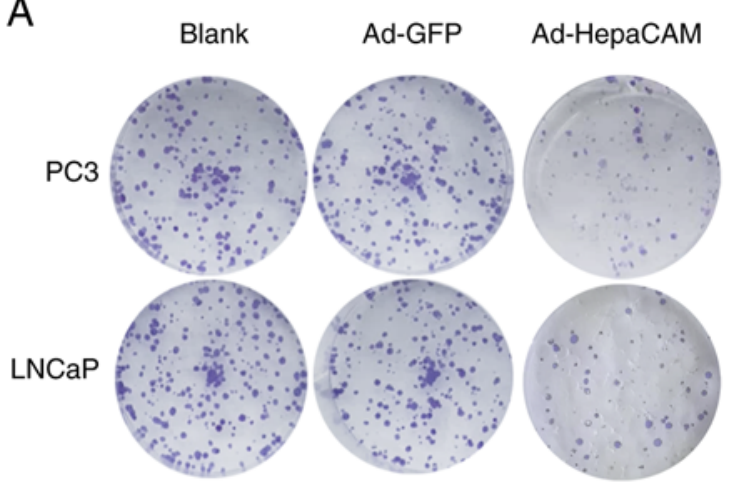

C

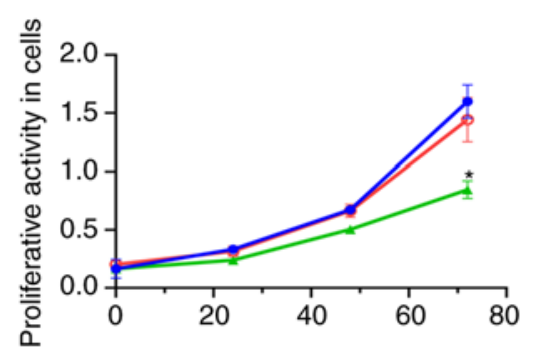

B

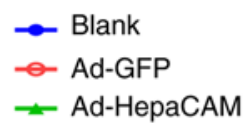

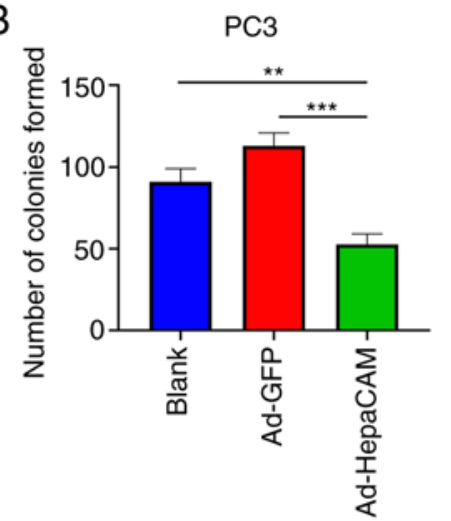
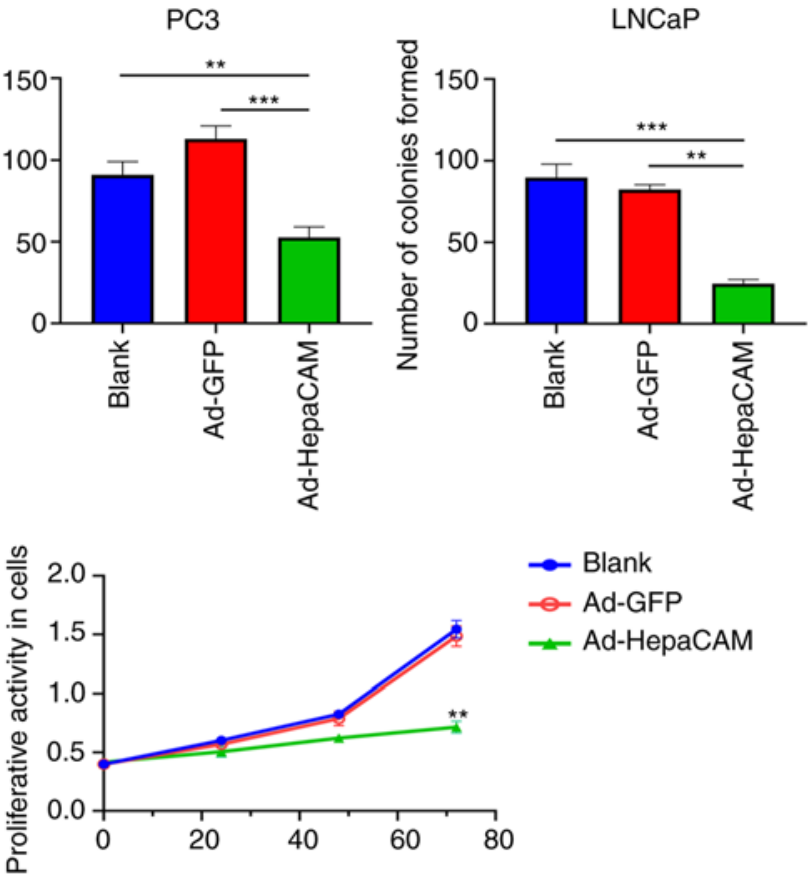
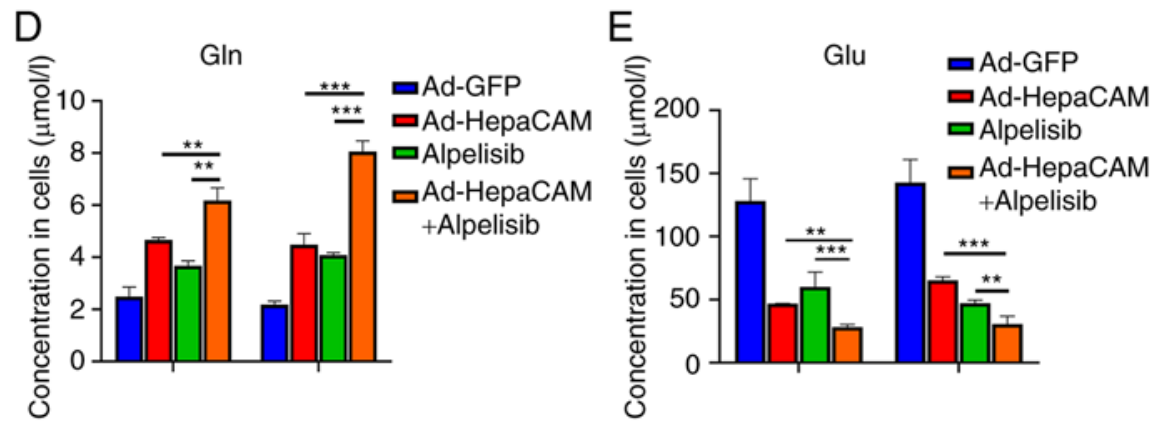

$\mathrm{F}$

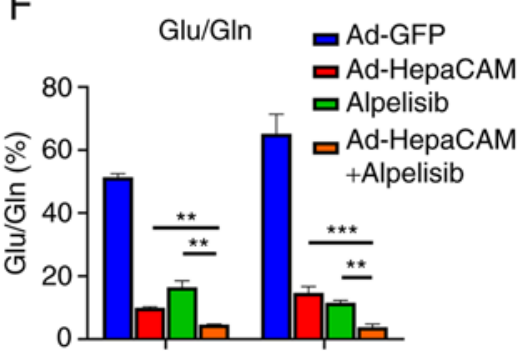

Figure 4. Overexpression of HepaCAM inhibits glutamine metabolism reprogramming and proliferation in PCa cells. (A) Colony formation assay and (B) statistical analysis of the numbers of colonies in the cells after the treatment of Ad-HepaCAM. (C) The proliferative activity in cells after the treatment of Ad-HepaCAM by CCK-8 assay. (D-F) Mass spectrometry tested the concentration of (D) Gln and (E) Glu in the PCa cells after the treatment of Ad-HepaCAM or/and alpelisib, and (F) comparison of the ratio of Glu to Gln. Data are represented as mean $\pm \mathrm{SD}$ of three individual experiments. ${ }^{*} \mathrm{P}<0.05,{ }^{* *} \mathrm{P}<0.01$, and ${ }^{* * *} \mathrm{P}<0.001$ vs. controls. HepaCAM, hepatocellular cell adhesion molecule; PCa, prostate cancer; Gln, glutamine; Glu, glutamate.

that metabolic changes in Gln occurred in patients with $\mathrm{PCa}$, which indicated the potential clinical significance of using Gln as a marker for clinical PCa screening.

High negative correlation between HepaCAM and PIK3CA expression in clinical PCa samples. The expression levels of HepaCAM and PIK3CA in $17 \mathrm{BPH}$ and 44 PCa tissue samples were measured using IHC staining. The results showed that the positive expression rate of HepaCAM reached 34.09\% (15/44) among the PCa samples, compared with $76.47 \%(13 / 17)$ in the BPH samples (Fig. 2I). In addition, the positive expression rate of PIK3CA was $86.36 \%$ (38/44) in PCa compared with $64.71 \%(11 / 17)$ in the BPH samples (Fig. 2J). Subsequently, HepaCAM and PIK3CA expression were found to be significantly correlated with the Gleason grade (Fig. 2E-H; Table II). Semi-quantitative staining scores revealed significantly decreased HepaCAM expression (P<0.05; Fig. 2I) and significantly increased PIK3CA expression ( $\mathrm{P}<0.01$; Fig. 2J) in the PCa tissues compared with those in the BPH tissues. Spearman's correlation analysis calculated that the expression of HepaCAM was negatively correlated with that of PIK3CA ( $\mathrm{P}<0.0001$; Fig. 2K).

Overexpression of HepaCAM inhibits the expression of PIK3CA in PCa cells. The expression of HepaCAM and PIK3CA in normal prostate epithelial cells (RWPE-1) compared with PCa cell lines PC3 and LNCaP were next compared in vitro. As shown in Fig. $3 \mathrm{~A}$ and $\mathrm{B}$, both mRNA and protein expression levels of HepaCAM in the cancer cell lines were significantly lower compared with those in the RWPE-1 cells, while the expression levels of PIK3CA were significantly higher in the cancer cell lines $(\mathrm{P}<0.05)$. To ultimately establish the role of HepaCAM in Gln metabolism and proliferation in the PCa cells, Ad-HepaCAM was first transfected into the PC3 and LNCaP cells. The results revealed significantly increased expression levels of HepaCAM in the PC3 and LNCaP cells in the Ad-HepaCAM group compared with those in the Ad-GFP group at both the mRNA and protein levels $(\mathrm{P}<0.001$; Fig. 3C-E). In addition, the expression of PIK3CA in both PC3 and LNCaP cells was significantly 
A

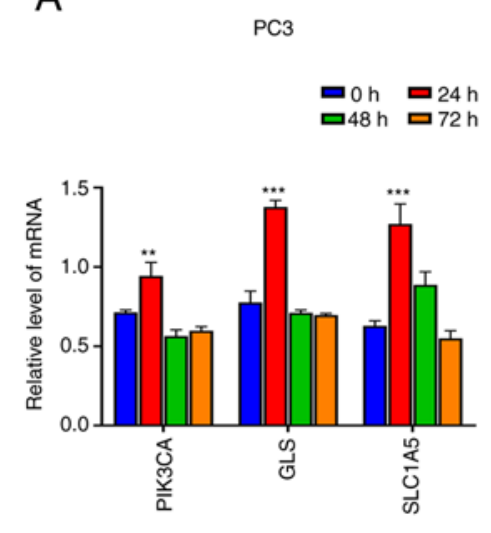

C
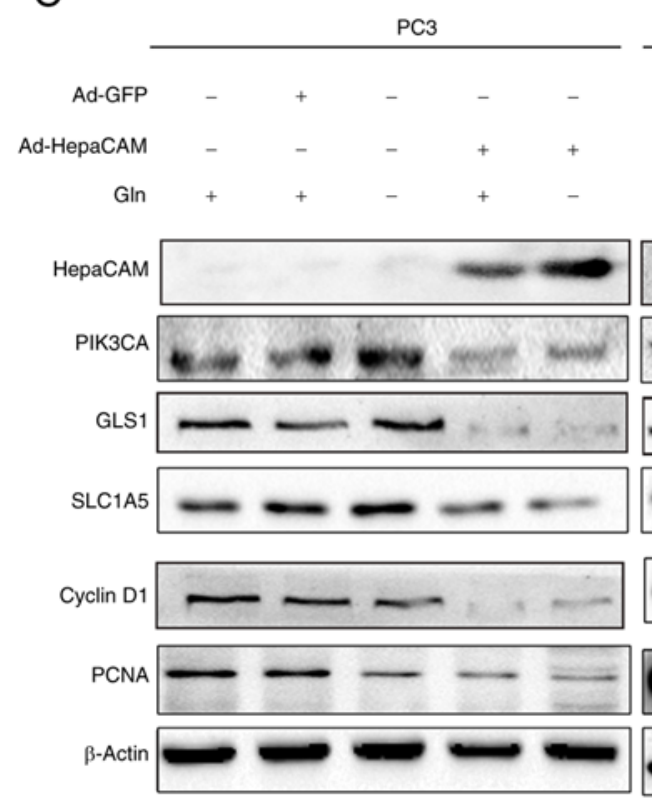

B
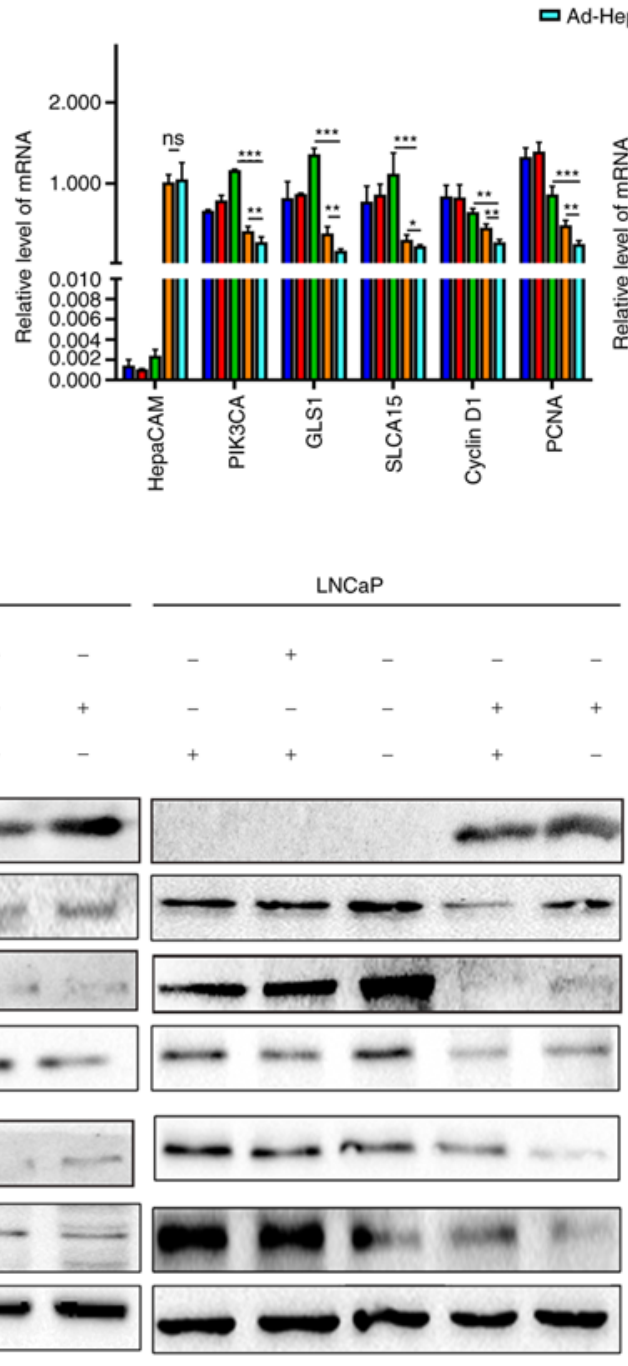

ש+Gln ad-GFP

口-GIn 口Ad-HepaCAM LNCaP HepaCAM/-Gin
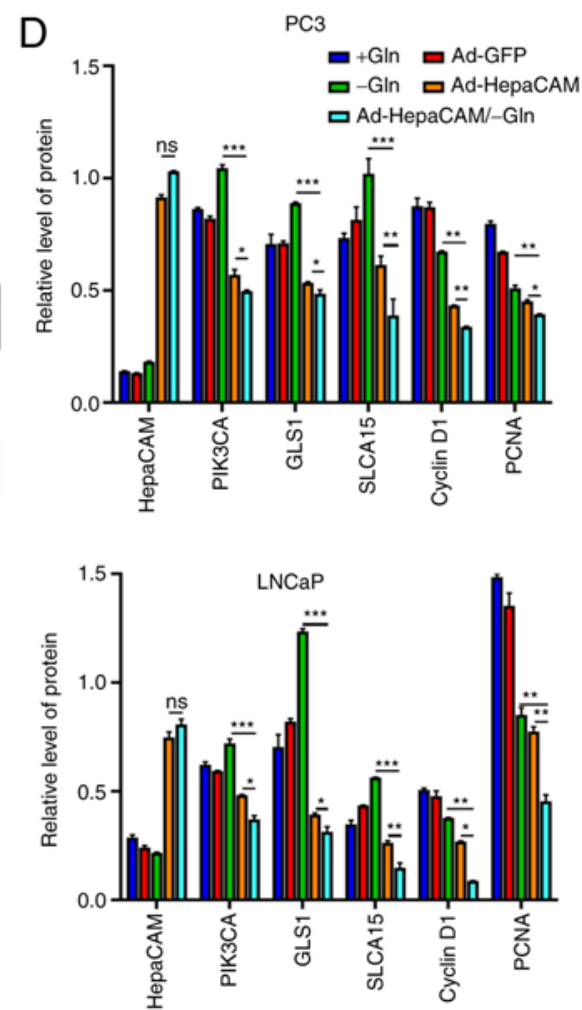

Figure 5. Overexpression of HepaCAM reverses stress resistance to reprogramming of glutamine metabolism in PCa cells. (A) mRNA expression levels of PIK3CA, GLS and SLC1A5 were measured at different time points after Gln deprivation by RT-qPCR. ${ }^{* *} \mathrm{P}<0.01$, and ${ }^{* * * *} \mathrm{P}<0.001 \mathrm{vs.} 0 \mathrm{~h}$. (B) RT-qPCR detection of mRNA levels of HepaCAM, PIK3CA, GLS, SLC1A5, Cyclin D1, and PCNA in PC3 and LNCaP cells after Ad-HepaCAM or Gln deprivation (24 h). $(\mathrm{C}$ and $\mathrm{D})$ Western blot analysis and quantification of the protein expression of the molecules mentioned in (B). $\beta$-actin were used as internal controls. Data are represented as mean $\pm \mathrm{SD}$ of three individual experiments. ${ }^{*} \mathrm{P}<0.05,{ }^{* *} \mathrm{P}<0.01$, and ${ }^{* * *} \mathrm{P}<0.001$; ns, not significant. HepaCAM, hepatocellular cell adhesion molecule; PIK3CA, phosphatidylinositol-4,5-bisphosphate 3-kinase catalytic subunit $\alpha$; GLS, glutaminase; SLC1A5, solute carrier family 1 member 5; PCNA, proliferating cell nuclear antigen.

downregulated by the overexpression of HepaCAM $(\mathrm{P}<0.01$; Fig. 3C-E).

Overexpression of HepaCAM inhibits Gln metabolism reprogramming and proliferation in PCa cells. Subsequently, the present study next investigated whether overexpression of HepaCAM can mediate an impact on Gln metabolism reprogramming and $\mathrm{PCa}$ cell proliferation. The results revealed that compared with that in the blank and Ad-GFP groups, the expression in PC3 and LNCaP cells of Gln metabolism components GLS and SLC1A5 in addition to cyclin D1 and PCNA, proteins associated with proliferation, were significantly reduced in the Ad-HepaCAM group at the mRNA and protein levels ( $\mathrm{P}<0.05$; Fig. 3F-H). Consistently, the results showed that the overexpression of HepaCAM also significantly inhibited the proliferation of $\mathrm{PCa}$ cells according to colony formation and CCK-8 assays compared with those in the Ad-GFP group $(\mathrm{P}<0.05$; Fig. 4A-C). MS analysis in PCa cells revealed that Gln (Fig. 4D) levels in the Ad-HepaCAM group were higher compared with those in the Ad-GFP group, while the levels of Glu were lower (Fig. 4E), rendering the ratio of Glu/Gln to be lower as a result of the overexpression of HepaCAM (Fig. 4F). This suggest that Gln metabolism reprogramming was inhibited. These results suggest that HepaCAM overexpression can inhibit Gln metabolism reprogramming and proliferation in PCa cells. 

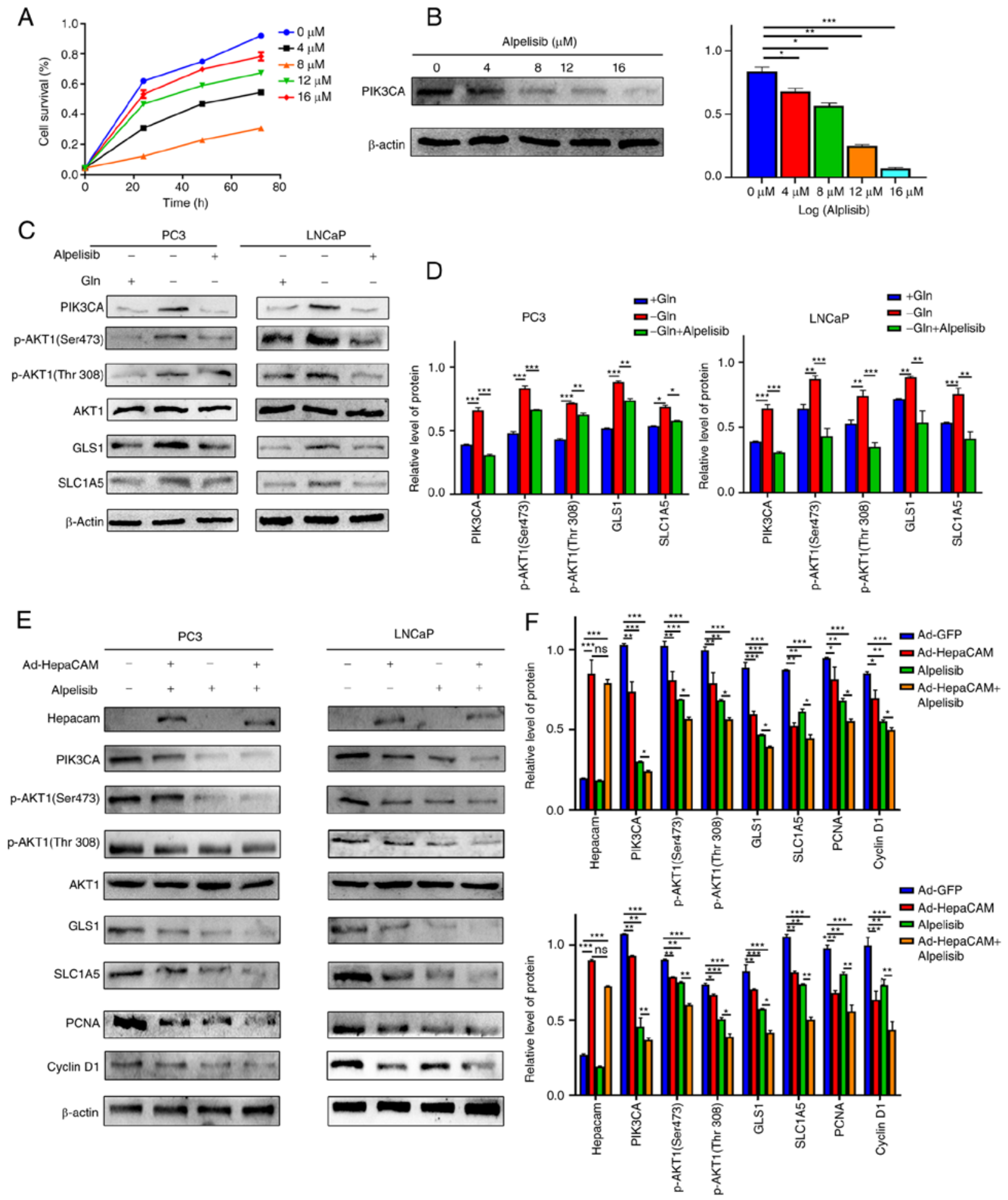

Figure 6. HepaCAM suppresses expression of glutamine metabolism-related and proliferation-related molecules by modulating PIK3CA. (A) CCK-8 assay was used to detect the toxicity of different concentrations of alpelisib $(0,4,8,12$ and $16 \mu \mathrm{M})$ on PC3 cells. (B) Western blot analysis was used to assess the inhibitory effect on PIK3CA expression with the concentrations of alpelisib used in A. (C and D) Western blot analysis was used to detect and analyse the protein expression of PIK3CA, p-AKT1(Ser473/Thr308), AKT1, GLS1, SLC1A5 after Gln deprivation or/and alpelisib treatment in PC3 and LNCaP cells (8 $\mu$ M, 24 h). (E and F) Western blot analysis was used to detect and analyse the protein expression of HepaCAM, cyclin D1, PCNA and the molecules mentioned in $\mathrm{C}$ after Gln deprivation for 24 , then treatment with Ad-HepaCAM or/and alpelisib for $72 \mathrm{~h}$. $\beta$-actin was used as internal controls. Data are represented as mean \pm SD of three individual experiments. ${ }^{\prime} \mathrm{P}<0.05,{ }^{* *} \mathrm{P}<0.01$, and ${ }^{* * * *} \mathrm{P}<0.001$ vs. controls. HepaCAM, hepatocellular cell adhesion molecule; PIK3CA, phosphatidylinositol-4,5-bisphosphate 3-kinase catalytic subunit $\alpha$; GLS, glutaminase; SLC1A5, solute carrier family 1 member 5; PCNA, proliferating cell nuclear antigen; Gln, glutamine.

Stress resistance by Gln metabolism can be reversed by HepaCAM overexpression. Despite the clear inhibitory effects on cell proliferation incurred by limited Gln levels, cancer cells have the capacity to adapt to the conditions to promote survival and sustain proliferative functions (42). Measuring the expression of enzymes associated with 
A

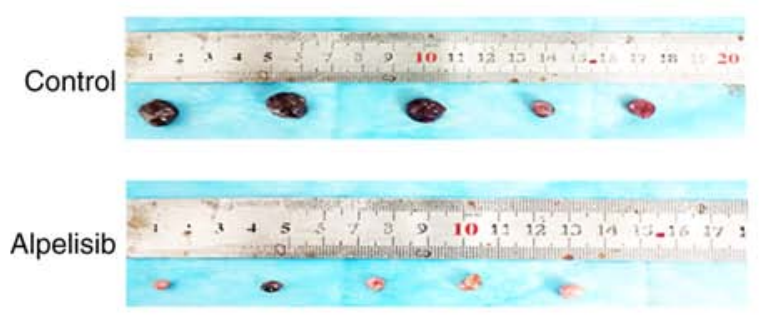

B

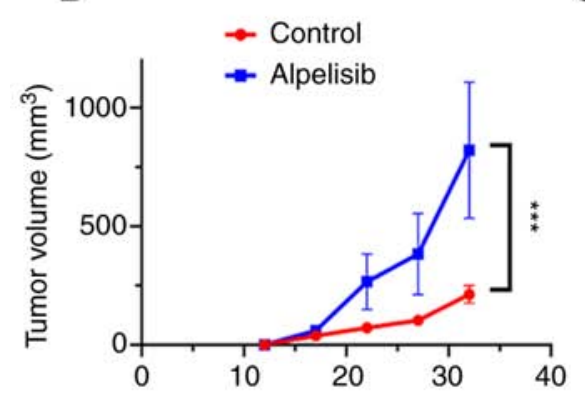

C

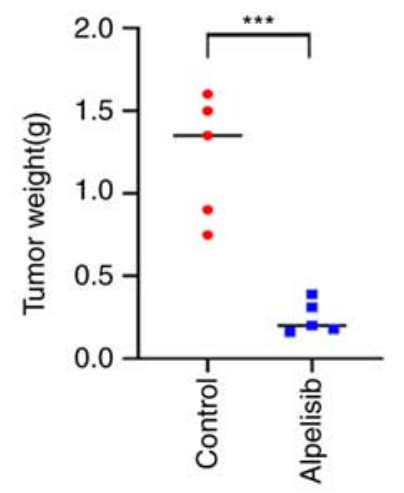

D
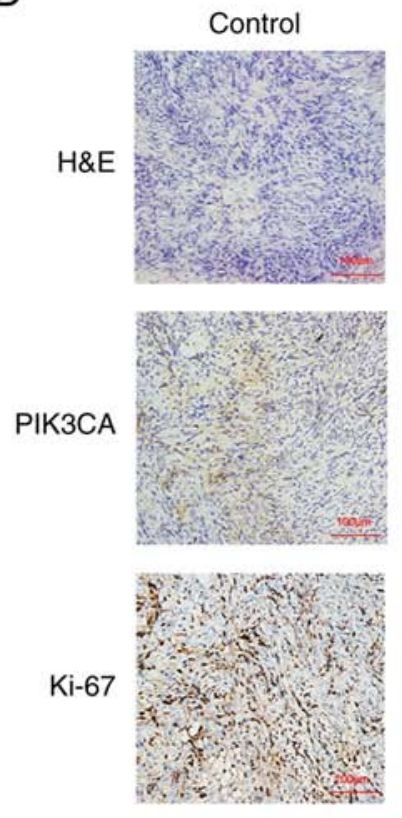

Alpelisib
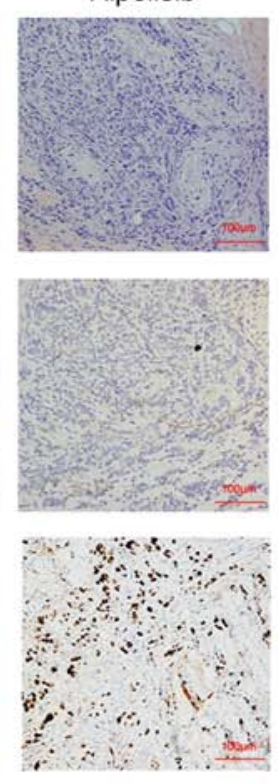

Figure 7. (A-C) Alpelisib significantly suppressed xenograft tumour grow in vivo. (D) IHC staining for the expression of PIK3CA and Ki67 in tumour tissue in the alpelisib group and matched group. Magnification, x100. PIK3CA, phosphatidylinositol-4,5-bisphosphate 3 -kinase catalytic subunit $\alpha$. ${ }^{* * *} \mathrm{P}<0.001$ vs. controls.

metabolism revealed that the expression of PIK3CA, GLS and SLC1A5 were significantly upregulated immediately after Gln deprivation for $24 \mathrm{~h}(\mathrm{P}<0.05$; Fig. 5A). Next, to explore the effects of HepaCAM on this positive response to nutrient starvation in PCa cells, particularly to Gln depletion, Ad-HepaCAM transfection was combined with Gln deprivation for $24 \mathrm{~h}$ alongside the corresponding control groups. RT-qPCR and western blotting analyses found that the overexpression of HepaCAM could reverse the upregulation of PIK3CA, GLS and SLC1A5 expression after Gln deprivation. Simultaneously, HepaCAM overexpression was found to significantly reduce the expression of proliferation-associated proteins cyclin D1 and PCNA ( $\mathrm{P}<0.05$; Fig. 5B-D). These results suggest that the overexpression of HepaCAM can reverse the metabolic stress resistance of PCa cells after Gln deprivation.

Stress resistance of Gln metabolism is mediated by PIK3CA. To ascertain whether the stress resistance demonstrated by Gln metabolism in PCa cells in response to a poor nutritional environment is associated with PIK3CA, alpelisib was used to suppress and inhibit PIK3CA expression. The minimum inhibitory concentration of cell viability exerted by alpelisib $(8 \mu \mathrm{M})$ was first determined using CCK- 8 assay $(\mathrm{P}<0.05$; Fig. 6A), while the trend of dose-independent inhibition was demonstrated according to western blotting results $(\mathrm{P}<0.05$; Fig. 6B). It was subsequently discovered that the stress resistance of Gln metabolism in PCa cells was reversed after PIK3CA expression was inhibited by alpelisib under Gln deprivation ( $\mathrm{P}<0.05$; Fig. $6 \mathrm{C}$ and $\mathrm{D})$. To verify the direct potentiating effects of PIK3CA on maintaining the stress resistance of Gln metabolism in vivo, $\mathrm{LNCaP}$ cells were subcutaneous injected into nude mice. In total, five mice were subcutaneously injected with alpelisib before the extent of tumour growth was measured over time. Alpelisib in the dosing group was found to significantly inhibit the growth and reduce the weight of xenograft tumours compared with those in the control group $(\mathrm{P}<0.001$; Fig. 7A-C). In addition, the expression levels of PIK3CA and Ki-67 in the tumour tissues of mice were suppressed by alpelisib (Fig. 7D). In conclusion, these results suggest that PIK3CA can mediate the stress resistance of Gln metabolism in PCa cells.

HepaCAM can suppress Gln metabolism reprogramming and cell proliferation of PCa by inhibiting PIK3CA expression. The present study next examined whether HepaCAM can regulate Gln metabolism reprogramming in PCa by regulating PIK3CA expression. To evaluate this hypothesis, Gln-deprived cells were treated with alpelisib and/or transfected with Ad-HepaCAM. The results demonstrated that the levels of PIK3CA protein expression and its downstream effector p-AKT1 (Ser473/Thr308), Gln metabolism components GLS1 and SLC1A5, proliferation-associated molecules cyclin D1 and PCNA were all significantly lower in the Ad-HepaCAM or alpelisib groups compared with those in their corresponding control groups. In particular, the inhibitory effects mediated by HepaCAM overexpression combined with alpelisib treatment were the most potent $(\mathrm{P}<0.05 ; \mathrm{Fig}$. $6 \mathrm{E}$ and $\mathrm{F})$. In addition, suppression of PIK3CA expression did not exert any significant effects on HepaCAM expression (Fig. 6E and F).

Next, CCK-8 assays were performed to detect the sensitivity of PC3 and LNCaP cells to alpelisib after Ad-HepaCAM transfection. The data revealed that alpelisib sensitivity was significantly increased in both cell lines after the overexpression of HepaCAM compared with that in the Ad-GFP group $(\mathrm{P}<0.05$, Fig. 8A). Furthermore, the results showed that the positive expression index of $\mathrm{Ki}-67$, a marker of cancer cell proliferation activity, was significantly 
A
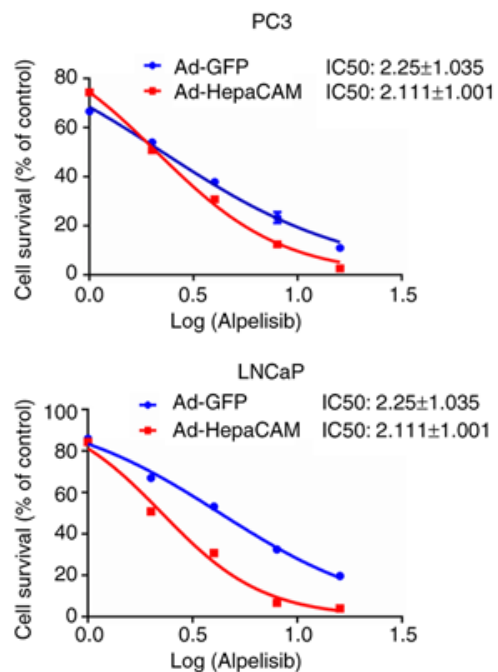

C

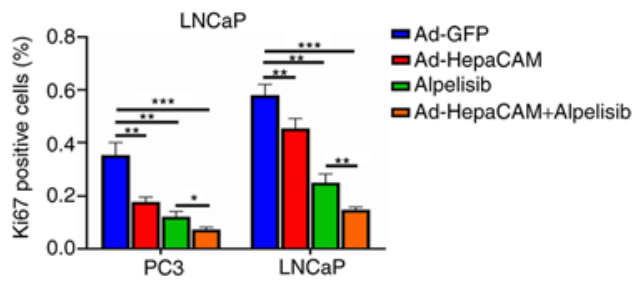

PC3
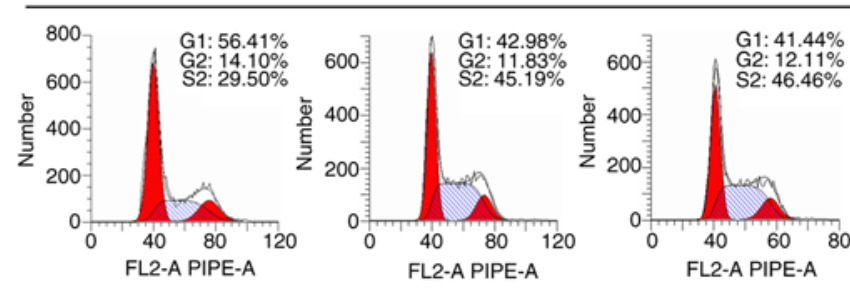

LNCaP
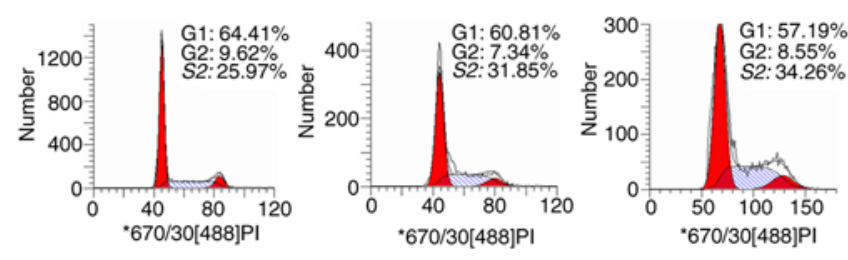
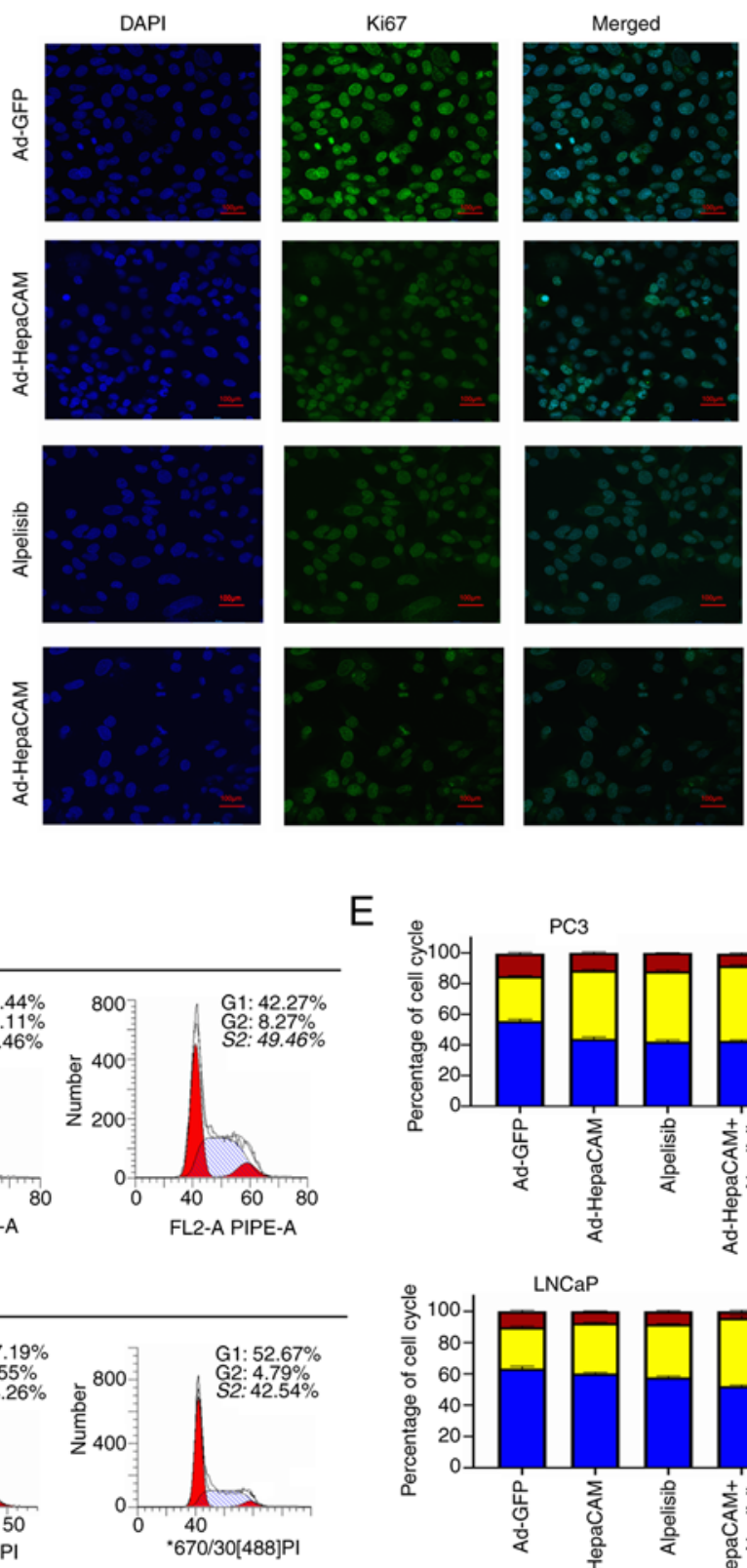

E
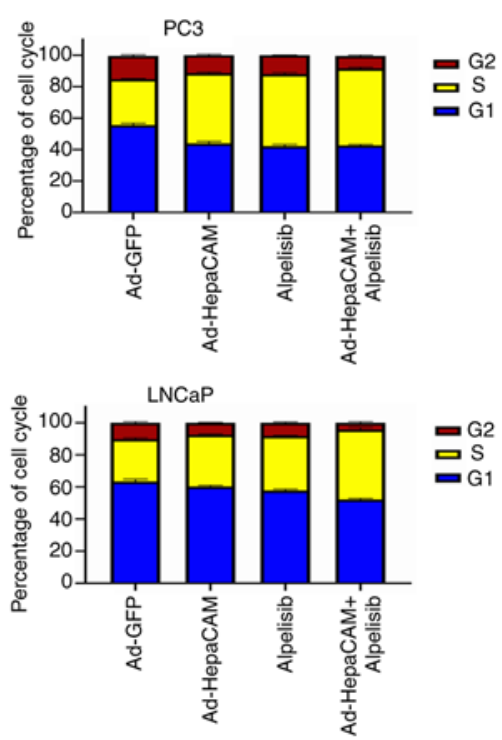

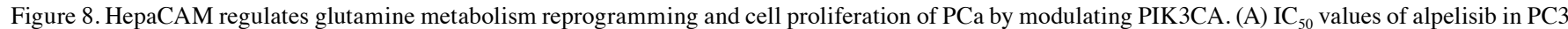
and LNCaP cells were measured by CCK-8 assay in Ad-GFP or Ad-HepaCAM groups ( $450 \mathrm{~nm}, 72 \mathrm{~h}$ ). (B) Ki-67 nuclear expression and (C) positive index in PC 3 and LNCaP cells were detected by IF after the treatment of Ad-HepaCAM or/and alpelisib. ${ }^{*} \mathrm{P}<0.05,{ }^{* *} \mathrm{P}<0.01$, and ${ }^{* * * *} \mathrm{P}<0.001$. (D) Flow cytometry detected cell cycle distribution after cells were cultured with Ad-HepaCAM or/and alpelisib, and (E) statistical analysis. Data are represented as mean \pm SD of three individual experiments. HepaCAM, hepatocellular cell adhesion molecule; $\mathrm{PCa}$, prostate cancer; $\mathrm{IC}_{50}$, half maximal inhibitory concentration.

lower compared with that in the Ad-GFP group following Ad-HepaCAM transfection and/or alpelisib, where the effects exerted by the combination of both treatments were also satisfactory $(\mathrm{P}<0.05$; Fig. $8 \mathrm{~B}$ and $\mathrm{C})$. Flow cytometry assay revealed that the proportion of cells in the $S$ phase was increased after transfection with Ad-HepaCAM and alpelisib (Fig. 8D and E). By contrast, MS results revealed that the combination of alpelisib and HepaCAM overexpression increased the concentration of Gln further in the PCa cells while suppressing its conversion to Glu ( $\mathrm{P}<0.05$; Fig. 4D-F). These results suggest that HepaCAM overexpression can inhibit Gln metabolic reprogramming in PCa cells through
PIK3CA to reverse their stress resistance, which in turn inhibits cell proliferation and tumour growth.

\section{Discussion}

In addition to essential sugars and fatty acids, glutamine (Gln) is another important source of nutrients that can accelerate the mitochondrial Kreb's cycle, in a process known as glutaminolysis, which involves the catabolism of Gln into glutamate (Glu), which is then metabolized into $\alpha$-ketoglutarate to support ATP production by feeding into the Kreb's cycle (5). In certain cases, cancer cells can reprogram themselves to 


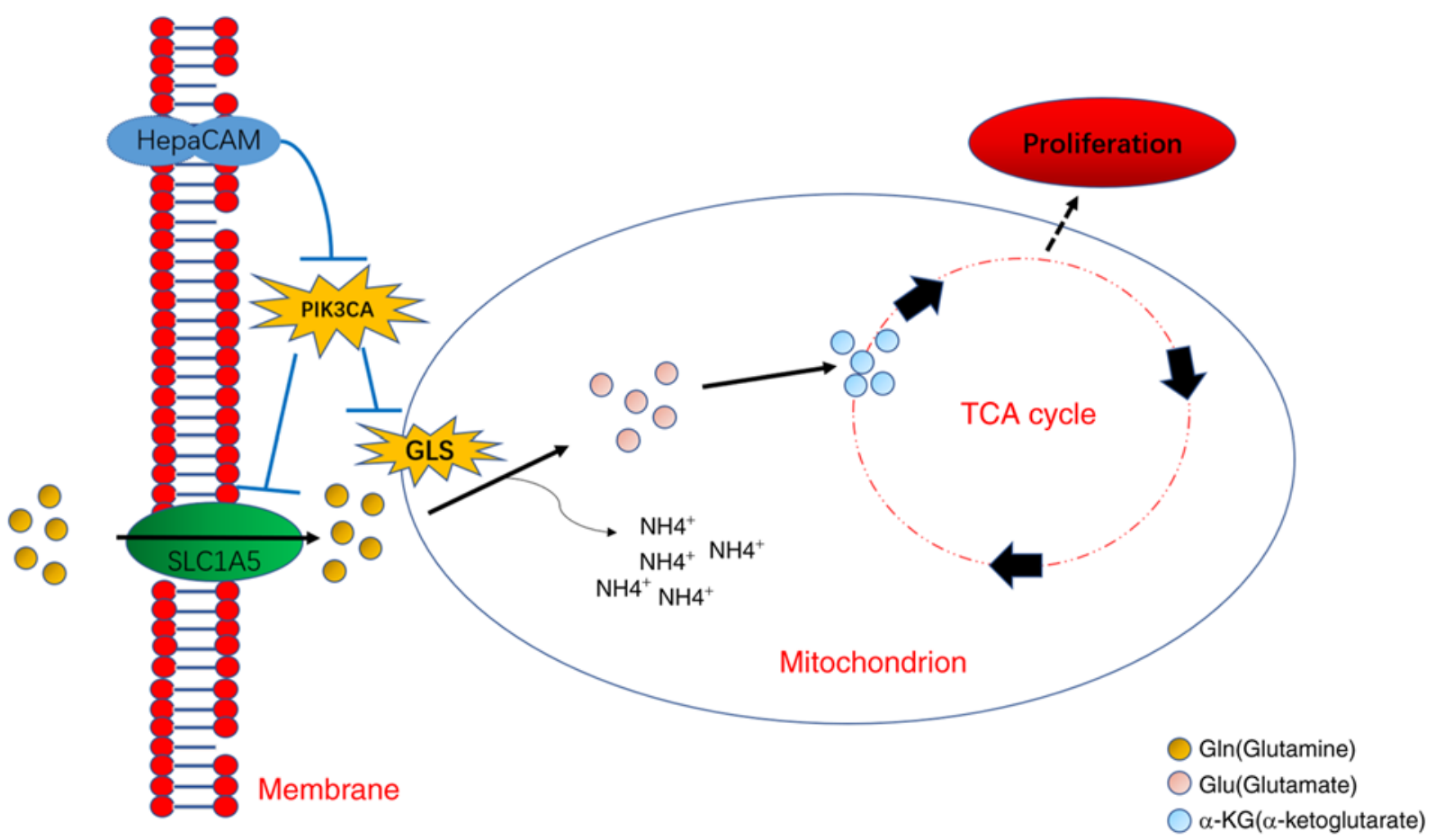

Figure 9. The mechanism of HepaCAM in regards to the inhibition of glutamine metabolism of PCa. HepaCAM inhibits glutamine metabolism-related molecules GLS1, SLC1A5 expression by PIK3CA signalling and reduces transportation and decomposition of glutamine, thereby inhibiting the proliferation driven by the tricarboxylic acid cycle (TCA) cycle. HepaCAM, hepatocellular cell adhesion molecule; PCa, prostate cancer; PIK3CA, phosphatidylinositol-4,5-bisphosphate 3-kinase catalytic subunit $\alpha$; GLS, glutaminase; SLC1A5, solute carrier family 1 member 5.

become dependent on certain metabolic pathways, activities or processes, such that the cell adopts a type of oncogenic addiction, in a phenomenon known as 'metabolic reprogramming' (6). Importantly, this type of metabolic reprogramming can be tissue-specific, and in context, downstream of both genetic and environmental factors, such that it can present vulnerabilities that can be therapeutically exploited $(43,44)$. Therefore, the present study compared the levels of various amino acids in the blood samples of normal individuals and patients with prostate cancer (PCa). Gln levels exhibited one of the most sensitive and significant differences, which was in turn correlated with the Gleason score based on the clinical parameters of the patients. In addition, through the detection and analysis of the levels of Gln and metabolic flux in the blood samples and PCa cells, it was found that Gln metabolism reprogramming was also common among patients with $\mathrm{PCa}$. Therefore, it was hypothesized that Gln levels have potential as a type of auxiliary diagnostic marker for $\mathrm{PCa}$ in a clinical setting.

Previous studies reported that hepatocellular cell adhesion molecule (HepaCAM) can serve a significant role in tumour cell proliferation, apoptosis, migration and invasion, since its expression is typically absent in cancer tissues $(19,45)$. In addition, previous reports have found that mutation in phosphatidylinositol-4,5-bisphosphate 3-kinase catalytic subunit $\alpha$ (PIK3CA) can activate PI3K/AKT signalling downstream to induce alterations in Gln metabolism in tumour cells (29). In the present study, bioinformatics analyses of various databases were performed to predict the correlation in the expression levels between HepaCAM and PIK3CA. These data revealed that PIK3CA and that of glutaminase (GLS) and as solute carrier family 1 member 5 (SLC1A5), components of Gln metabolism, are expressed at high levels in PCa tissues, all of which in turn correspond to poorer prognoses. In addition, a negative correlation was found between HepaCAM and PIK3CA expression in PCa tissues as determined by IHC. Subsequently, HepaCAM and PIK3CA expression were found to be significantly correlated with the Gleason grade, which implies the different prognoses associated with their expression levels in PCa tissues. Therefore, these findings suggest that HepaCAM can regulate Gln metabolic reprogramming in PCa by regulating PIK3CA expression.

At present, the most extensively studied metabolic alteration in cancer cells is their high reliance on glycolysis even under sufficient oxygen supply to sustain oxidative phosphorylation in the mitochondria, in a process known as the Warburg effect (19). The rapid ATP production as a result of this glycolytic upregulation is considered to potentiate the proliferation of cancer cells (46). However, it has been previously reported that the catabolic and anabolic roles of both glucose and Gln can cooperate in promoting tumour growth (47). A previous study found that HepaCAM can inhibit the Warburg effect in renal cell carcinoma cells (19). Therefore, it could be hypothesized that HepaCAM can inhibit Gln metabolism reprogramming in PCa. In the present study, the mRNA and protein expression levels of HepaCAM and PIK3CA in normal prostate epithelial cells RWPE-1 and prostate cancer cell lines PC3 and LNCaP were next assessed. After the induced overexpression of HepaCAM in the cells lines, the expression of PIK3CA and Gln metabolism components GLS and SLC1A5, in addition to tumour proliferation-associated molecules PCNA and cyclin D1, were all reduced. These results suggest that the loss of HepaCAM expression in tumours may 
promote dysfunctions in Gln metabolism, promote cell proliferation and tumour development.

Subsequently, the results from the present study revealed stress resistance or a compensatory effect in Gln metabolism in PCa cells following Gln starvation. GLS and SLC1A5 expression were temporarily increased in the short-term after Gln deprivation in cell culture conditions, which may be a potential cause of the ineffectiveness of Gln starvation in PCa. On this basis, HepaCAM was overexpressed, which eliminated this stress compensation effect. In addition, the inhibitory effects of HepaCAM combined with Gln deprivation on tumour proliferation were significantly more potent compared with those mediated by either treatment alone. Although this compensatory response has been previously reported in cervical cancer tissues, a previous study found this effect after treatment with inhibitors of GLS1 (48). In addition, another previous study discovered that arginine can participate in the mediation of this type of compensation (49). Since abnormal arginine levels were also found in the blood samples of patients with $\mathrm{PCa}$ in the present study, the possibility of other signalling pathways mediating this type of stress compensation apart from HepaCAM cannot be ruled out.

The present study then explored the effects of HepaCAM on Gln metabolism reprogramming of $\mathrm{PCa}$. As one of the catalytic subunits of classical PI3K, PIK3CA serves a key role in activating the PI3K/AKT signalling pathway (25). A number of studies have previously found that PIK3CA has a high frequency of mutations in tumours $(40,41,50)$. Through bioinformatics analysis, it was found that PIK3CA primarily serves a facilitating role in PCa pathophysiology, where it was frequently found to be activated by amplification. This suggests that this form of PIK3CA activation is tissue-specific. Subsequently, higher PIK3CA expression levels were also found in $\mathrm{PCa}$ tissues compared with those in the adjacent normal tissues, where they in turn were correlated inversely and significantly with those of HepaCAM. Alpelisib is a specific inhibitor of PIK3CA (51). The present study found that inhibition of PIK3CA expression by alpelisib significantly reversed the stress resistance of PCa cells to Gln deprivation, supporting the possible effects of PIK3CA in this phenomenon. After the PCa cells deprived of Gln for $24 \mathrm{~h}$ were treated with alpelisib and transfected with Ad-HepaCAM at the same time, the phosphorylation of AKT, the expression of Gln metabolism-associated and proliferation-associated proteins were all reduced. In addition, the suppression of PIK3CA exerted no significant impact on HepaCAM expression. These observations suggest that HepaCAM can strengthen the inhibition of Gln metabolic reprogramming and proliferation in $\mathrm{PCa}$ by reversing stress resistance through inhibiting PIK3CA expression. However, the present study did not assess the possibility of a direct interaction between HepaCAM and PIK3CA at the protein level. Instead, the protein structure database STRING was used to predict protein interaction, showing that HepaCAM may bind to PIK3CA directly. However, this requires further experimental verification.

Alpelisib is a PIK3CA inhibitor that has demonstrated antitumor activity in several types of cancer, including breast cancer (52) and epithelial ovarian cancer (53). In addition, it has been previously applied in clinical trials for the treatment of multiple tumours. Results from the present study suggest that it exerts significant inhibitory effects on PIK3CA in PCa cells in a dose-dependent manner. Although HepaCAM was shown to inhibit Gln metabolism reprogramming and proliferation in $\mathrm{PCa}$, the addition of alpelisib was able to significantly potentiate the inhibition caused by HepaCAM overexpression. Although a specific agonist of HepaCAM has not yet been successfully developed, the use of PIK3CA inhibitors to block tumour growth and cell proliferation appear to more likely result in greater clinical significance, which provides a valuable reference for the clinical treatment of PCa.

The present study has a number of limitations. Although the mRNA expression data of HepaCAM in the bioinformatics analysis is the relative expression value obtained after standardization according to the FPKM algorithm, the abundance value of HepaCAM expression in the TCGA PCa datasets were relatively low, which could have caused a certain degree of interference in the follow-up IHC experimental conclusions.

In summary, the present study uncovered abnormal Gln concentrations, which may be exploited as a potential indicator for the diagnosis of PCa. In addition, to the best of our knowledge, the present study was the first to discover that the HepaCAM/PIK3CA axis may be implicated in Gln metabolism reprogramming and $\mathrm{PCa}$ proliferation (Fig. 9). Mechanistically, HepaCAM overexpression reversed the stress resistance shown by Gln metabolism by downregulating PIK3CA expression. Importantly, the combination of PIK3CA inhibitor and overexpression of HepaCAM was able to effectively inhibit Gln metabolism reprogramming and $\mathrm{PCa}$ proliferation, thereby providing a certain clinical value for the treatment of $\mathrm{PCa}$.

\section{Acknowledgements}

The authors would like to thank Dr Chaowen Yu, Center for Clinical Molecular Medicine, Children's Hospital of Chongqing Medical University. Chongqing, China, for providing the technical assistance with the liquid chromatography-tandem mass spectrometry and gas chromatography-mass spectrometry assays.

\section{Funding}

The present study was supported by grants from the National Natural Science Foundation of China (grant no. 81272572).

\section{Availability of data and materials}

The datasets used and/or analysed during the current study are available from the corresponding author on reasonable request.

\section{Authors' contributions}

ZH, CL and YG designed the experiments. ZH, YG, TL and CY collected the specimens and analysed the clinical data. ZH, YG , TL and CY performed the experiments. CL and LO provided technical support in this research project and supervised the progress of the experiments. $\mathrm{ZH}$ and $\mathrm{YG}$ analysed the statistical data. ZH, LO and TL assembled and installed the figures. $\mathrm{ZH}, \mathrm{YG}$ and TL confirm the authenticity of all the raw data. All authors have read and approved the final manuscript for publication. 


\section{Ethics approval and consent to participate}

The present study was approved by the Ethics Committee of Chongqing Medical University (2016.12.16 Chongqing, China). Informed consent was obtained from the patients or their family members who agreed to the use of their samples in this study. The animal study was performed in accordance with the Animal Research Advisory Committee (ARAC) Guidelines and was approved by the Ethics Committee of Chongqing Medical University (Chongqing, China).

\section{Patient consent for publication}

Not applicable.

\section{Competing interests}

The authors declare that they have no competing interests.

\section{References}

1. Bray F, Ferlay J, Soerjomataram I, Siegel RL, Torre LA and Jemal A: Global cancer statistics 2018: GLOBOCAN estimates of incidence and mortality worldwide for 36 cancers in 185 countries. CA Cancer J Clin 68: 394-424, 2018.

2. Fu ZT, Guo XL, Zhang SW, Zheng RS, Zeng HM, Chen R, Wang SM, Sun KX, Wei WW and He J: Statistical analysis of incidence and mortality of prostate cancer in China, 2015. Zhonghua Zhong Liu Za Zhi 42: 718-722, 2020 (In Chinese).

3. Li Z and Zhang H: Reprogramming of glucose, fatty acid and amino acid metabolism for cancer progression. Cell Mol Life Sci 73: 377-392, 2016

4. Hanahan D and Weinberg RA: Hallmarks of cancer: The next generation. Cell 144: 646-674, 2011.

5. Altman B, Stine Z and Dang C: From Krebs to clinic: Glutamine metabolism to cancer therapy. Nat Rev Cancer 16: 619-634, 2016.

6. Zhang J, Pavlova NN and Thompson CB: Cancer cell metabolism: The essential role of the nonessential amino acid, glutamine. EMBO J 36: 1302-1315, 2017.

7. Mates JM, Segura JA, Martin-Rufian M, Campos-Sandoval JA, Alonso FJ and Marquez J: Glutaminase isoenzymes as key regulators in metabolic and oxidative stress against cancer. Curr Mol Med 13: 514-534, 2013.

8. Hassanein M, Hoeksema MD, Shiota M, Qian J, Harris BK, Chen H, Clark JE, Alborn WE, Eisenberg R and Massion PP: SLC1A5 mediates glutamine transport required for lung cancer cell growth and survival. Clin Cancer Res 19: 560-570, 2013.

9. Strmiska V, Michalek P, Eckschlager T, Stiborova M, Adam V, Krizkova S and Heger Z: Prostate cancer-specific hallmarks of amino acids metabolism: Towards a paradigm of precision medicine. Biochim Biophys Acta Rev Cancer 1871: 248-258, 2019.

10. Martinez-Outschoorn U, Lisanti M and Sotgia F: Catabolic cancer-associated fibroblasts transfer energy and biomass to anabolic cancer cells, fueling tumor growth. Semin Cancer Biol 25: 47-60, 2014.

11. Huang $\mathrm{C}$ and McConathy $\mathrm{J}$ : Radiolabeled amino acids for oncologic imaging. J Nucl Med 54: 1007-1010, 2013.

12. Twum-Ampofo J, Fu D, Passaniti A, Hussain A and Siddiqui M: Metabolic targets for potential prostate cancer therapeutics. Curr Opin Oncol 28: 241-247, 2016.

13. Chung Moh M, Hoon Lee L and Shen S: Cloning and characterization of hepaCAM, a novel Ig-like cell adhesion molecule suppressed in human hepatocellular carcinoma. J Hepatol 42: 833-841, 2005.

14. Moh MC, Zhang C, Luo C, Lee LH and Shen S: Structural and functional analyses of a novel ig-like cell adhesion molecule, hepaCAM, in the human breast carcinoma MCF7 cells. J Biol Chem 280: 27366-27374, 2005.

15. Song X, Wang Y, Du H, Fan Y, Yang X, Wang X, Wu X and Luo C: Overexpression of HepaCAM inhibits cell viability and motility through suppressing nucleus translocation of androgen receptor and ERK signaling in prostate cancer. Prostate 74: 1023-1033, 2014.
16. Tang M, Zhao Y, Liu N, Chen E, Quan Z, Wu X and Luo C: Overexpression of HepaCAM inhibits bladder cancer cell proliferation and viability through the AKT/FoxO pathway. J Cancer Res Clin Oncol 143: 793-805, 2017.

17. Wang X, Chen E, Yang X, Wang Y, Quan Z, Wu X and Luo C: 5-azacytidine inhibits the proliferation of bladder cancer cells via reversal of the aberrant hypermethylation of the hepaCAM gene. Oncol Rep 35: 1375-1384, 2016.

18. Quan Z, He Y, Luo C, Xia Y,Zhao Y, Liu N and Wu X: Interleukin 6 induces cell proliferation of clear cell renal cell carcinoma by suppressing hepaCAM via the STAT3-dependent up-regulation of DNMT1 or DNMT3b. Cell Signal 32: 48-58, 2017.

19. Fan Y, Ou L, Fan J, Li L, Wu X, Luo C, Gao Y and Niu L: HepaCAM regulates warburg effect of renal cell carcinoma via HIF-1 $\alpha / N F-\kappa B$ signaling pathway. Urology 127: 61-67, 2019.

20. Deng Q, Luo L, Quan Z, Liu N, Du Z, Sun W, Luo C and Wu X: HepaCAM inhibits cell proliferation and invasion in prostate cancer by suppressing nuclear translocation of the androgen receptor via its cytoplasmic domain. Mol Med Rep 19: 2115-2124, 2019.

21. Yuan TL and Cantley LC: PI3K pathway alterations in cancer: Variations on a theme. Oncogene 27: 5497-5510, 2008.

22. Liu W, Yuan Y, Sun C, Balasubramanian B, Zhao Z and An L: Effects of dietary betaine on growth performance, digestive function, carcass traits, and meat quality in indigenous yellow-feathered broilers under long-term heat stress. Animals (Basel) 9: 506, 2019.

23. Liu WC, Guo Y, Zhao ZH, Jha R and Balasubramanian B: Algae-derived polysaccharides promote growth performance by improving antioxidant capacity and intestinal barrier function in broiler chickens. Front Vet Sci 7: 601336, 2020.

24. Guo Y, Zhao ZH, Pan ZY, An LL, Balasubramanian B and Liu WC: New insights into the role of dietary marine-derived polysaccharides on productive performance, egg quality, antioxidant capacity, and jejunal morphology in late-phase laying hens. Poult Sci 99: 2100-2107, 2020.

25. Hiles ID, Otsu M, Volinia S, Fry MJ, Gout I, Dhand R, Panayotou G, Ruiz-Larrea F, Thompson A, Totty NF, et al: Phosphatidylinositol 3-kinase: Structure and expression of the 110 kd catalytic subunit. Cell 70: 419-429, 1992.

26. Holst F, Werner HM, Mjøs S, Hoivik EA, Kusonmano K, Wik E, Berg A, Birkeland E, Gibson WJ, Halle MK, et al: PIK3CA amplification associates with aggressive phenotype but not markers of AKT-MTOR signaling in endometrial carcinoma. Clin Cancer Res 25: 334-345, 2019.

27. Han C, Yang L, Choi HH, Baddour J, Achreja A, Liu Y, Li Y, Li J, Wan G, Huang C, et al: Amplification of USP13 drives ovarian cancer metabolism. Nat Commun 7: 13525, 2016.

28. Hao Y, Samuels Y, Li Q, Krokowski D, Guan BJ, Wang C, Jin Z, Dong B, Cao B, Feng X, et al: Oncogenic PIK3CA mutations reprogram glutamine metabolism in colorectal cancer. Nat Commun 7: 11971, 2016.

29. Boku S, Watanabe M, Sukeno M, Yaoi T, Hirota K, Iizuka-Ohashi M, Itoh K and Sakai T: Deactivation of glutaminolysis sensitizes PIK3CA-mutated colorectal cancer cells to aspirin-induced growth inhibition. Cancers (Basel) 12: 1097, 2020.

30. Lau CE, Tredwell GD, Ellis JK, Lam EW and Keun H: Metabolomic characterisation of the effects of oncogenic PIK3CA transformation in a breast epithelial cell line. Sci Rep 7: 46079, 2017.

31. Du Z, Li L, Sun W, Wang X, Zhang Y, Chen Z, Yuan M, Quan Z, Liu N, Hao Y, et al: HepaCAM inhibits the malignant behavior of castration-resistant prostate cancer cells by downregulating Notch signaling and PF-3084014 (a $\gamma$-secretase inhibitor) partly reverses the resistance of refractory prostate cancer to docetaxel and enzalutamide in vitro. Int J Oncol 53: 99-112, 2018.

32. Zhao Y, Balasubramanian B, Guo Y, Qiu SJ, Jha R and Liu WC: Dietary enteromorpha polysaccharides supplementation improves breast muscle yield and is associated with modification of mrna transcriptome in broiler chickens. Front Vet Sci 8: 663988, 2021.

33. Guo Y, Balasubramanian B, Zhao ZH and Liu WC: Marine algal polysaccharides alleviate aflatoxin B1-induced bursa of Fabricius injury by regulating redox and apoptotic signaling pathway in broilers. Poult Sci 100: 844-857, 2021.

34. Liu WC, Ou BH, Liang ZL, Zhang R and Zhao ZH: Algae-derived polysaccharides supplementation ameliorates heat stress-induced impairment of bursa of Fabricius via modulating NF- $\kappa$ B signaling pathway in broilers. Poult Sci 100: 101139, 2021. 
35. Liu WC, Zhu YR, Zhao ZH, Jiang P and Yin FQ: Effects of dietary supplementation of algae-derived polysaccharides on morphology, tight junctions, antioxidant capacity and immune response of duodenum in broilers under heat stress. Animals (Basel) 11: 2279, 2021.

36. Yu C, Huang S, Wang M, Zhang J, Liu H, Yuan Z, Wang X, He X, Wang J and Zou L: A novel tandem mass spectrometry method for first-line screening of mainly beta-thalassemia from dried blood spots. J Proteomics 154: 78-84, 2017.

37. Geng HT, Cao RJ, Cheng L and Liu CY: Overexpression of hepatocyte cell adhesion molecule (hepaCAM) inhibits the proliferation, migration, and invasion in colorectal cancer cells. Oncol Res 25: 1039-1046, 2017.

38. Zhang QL, Luo CL, Wu XH, Wang CY, Xu X, Zhang YY, Liu Q and Shen SL: HepaCAM induces G1 phase arrest and promotes c-Myc degradation in human renal cell carcinoma. J Cell Biochem 112: 2910-2919, 2011.

39. Wise DR, DeBerardinis RJ, Mancuso A, Sayed N, Zhang XY, Pfeiffer HK, Nissim I, Daikhin E, Yudkoff M, McMahon SB and Thompson CB: Myc regulates a transcriptional program that stimulates mitochondrial glutaminolysis and leads to glutamine addiction. Proc Natl Acad Sci USA 105: 18782-18787, 2008.

40. Kandoth C, McLellan MD, Vandin F, Ye K, Niu B, Lu C, Xie M, Zhang Q, McMichael JF, Wyczalkowski MA, et al: Mutational landscape and significance across 12 major cancer types. Nature 502: 333-339, 2013

41. Lawrence MS, Stojanov P, Mermel CH, Robinson JT Garraway LA, Golub TR, Meyerson M, Gabriel SB, Lander ES and Getz G: Discovery and saturation analysis of cancer genes across 21 tumour types. Nature 505: 495-501, 2014.

42. Torre LA, Sauer AM, Chen MS Jr, Kagawa-Singer M, Jemal A and Siegel R: Cancer statistics for Asian Americans, Native Hawaiians, and Pacific Islanders, 2016: Converging incidence in males and females. CA Cancer J Clin 66: 182-202, 2016.

43. Mayers JR, Torrence ME, Danai LV, Papagiannakopoulos T, Davidson SM, Bauer MR, Lau AN, Ji BW, Dixit PD and Hosios AM: Tissue of origin dictates branched-chain amino acid metabolism in mutant Kras-driven cancers. Science 353: 1161-1165, 2016.

44. Yuneva MO, Fan TW, Allen TD, Higashi RM, Ferraris DV, Tsukamoto T, Matés JM, Alonso FJ, Wang C, Seo Y, et al: The metabolic profile of tumors depends on both the responsible genetic lesion and tissue type 15: 157-170, 2012.
45. Li T, Liu N, Gao Y, Quan Z, Hao Y, Yu C, Li L, Yuan M, Niu L, Luo $\mathrm{C}$ and $\mathrm{Wu} \mathrm{X}$ : Long noncoding RNA HOTAIR regulates the invasion and metastasis of prostate cancer by targeting hepaCAM. Br J Cancer 124: 247-258, 2020.

46. McGuirk S, Gravel S, Deblois G, Papadopoli DJ, Faubert B, Wegner A, Hiller K, Avizonis D, Akavia UD, Jones RG, et al: PGC-1 $\alpha$ supports glutamine metabolism in breast cancer. Cancer Metab 1: 22, 2013.

47. DeBerardinis RJ and Cheng T: Q's next: The diverse functions of glutamine in metabolism, cell biology and cancer. Oncogene 29: 313-324, 2010.

48. Biancur DE, Paulo JA, Małachowska B, Quiles Del Rey M, Sousa CM, Wang X, Sohn AS, Chu GC, Gygi SP, Harper JW, et al: Compensatory metabolic networks in pancreatic cancers upon perturbation of glutamine metabolism. Nat Commun 8: 15965, 2017.

49. Lowman XH, Hanse EA, Yang Y, Ishak Gabra MB, Tran TQ, $\mathrm{Li} \mathrm{H}$ and Kong M: p53 promotes cancer cell adaptation to glutamine deprivation by upregulating Slc7a3 to increase arginine uptake. Cell Rep 26: 3051-3060.e3054, 2019.

50. Jiang W,He T, Liu S, Zheng Y, Xiang L, Pei X, Wang Z and Yang H: The PIK3CA E542K and E545K mutations promote glycolysis and proliferation via induction of the $\beta$-catenin/SIRT3 signaling pathway in cervical cancer. J Hematol Oncol 11: 139, 2018.

51. André F, Ciruelos E, Rubovszky G, Campone M, Loibl S, Rugo HS, Iwata H, Conte P, Mayer IA, Kaufman B, et al: Alpelisib for PIK3CA-mutated, hormone receptor-positive advanced breast cancer. N Engl J Med 380: 1929-1940, 2019.

52. Juric D, Janku F, Rodón J, Burris HA, Mayer IA, Schuler M, Seggewiss-Bernhardt R, Gil-Martin M, Middleton MR, Baselga J, et al: Alpelisib plus fulvestrant in PIK3CA-altered and PIK3CA-wild-type estrogen receptor-positive advanced breast cancer: A phase 1b clinical trial. JAMA Oncol 5: e184475, 2019.

53. Konstantinopoulos PA, Barry WT, Birrer M, Westin SN, Cadoo KA, Shapiro GI, Mayer EL, O'Cearbhaill RE, Coleman RL, Kochupurakkal B, et al: Olaparib and $\alpha$-specific PI3K inhibitor alpelisib for patients with epithelial ovarian cancer: A dose-escalation and dose-expansion phase $1 \mathrm{~b}$ trial. Lancet Oncol 20: 570-580, 2019.

This work is licensed under a Creative Commons Attribution-NonCommercial-NoDerivatives 4.0 International (CC BY-NC-ND 4.0) License. 